\title{
Reflets
}

Revue ontaroise d'intervention sociale et communautaire

\section{Loi, homophobie et violence : légiférer pour punir les crimes motivés par la haine}

\section{Douglas Victor Janoff}

Volume 13, numéro 1, 2007

La violence dans tous ses états

URI : https://id.erudit.org/iderudit/016815ar

DOI : https://doi.org/10.7202/016815ar

Aller au sommaire du numéro

Éditeur(s)

Reflets : Revue ontaroise d'intervention sociale et communautaire

ISSN

1203-4576 (imprimé)

1712-8498 (numérique)

Découvrir la revue

Citer cet article

Janoff, D. (2007). Loi, homophobie et violence : légiférer pour punir les crimes motivés par la haine. Reflets, 13(1), 114-161. https://doi.org/10.7202/016815ar

Tous droits réservés (C) Reflets : Revue ontaroise d'intervention sociale et communautaire, 2007
Ce document est protégé par la loi sur le droit d'auteur. L'utilisation des services d'Érudit (y compris la reproduction) est assujettie à sa politique d'utilisation que vous pouvez consulter en ligne.

https://apropos.erudit.org/fr/usagers/politique-dutilisation/ 


\title{
Loi, homophobie et violence : légiférer pour punir les crimes motivés par la haine
}

\author{
Douglas Victor Janoff
}

\section{Résumé}

L'homophobie sous-tend de multiples pratiques juridiques qui minimisent l'importance de la violence envers les lesbiennes, gais, bisexuels et transsexuels (LGBT) ou l'excusent, voire qui nient son existence. Parfois, dans les cas d'erreurs de procédure ou d'administration, l'homophobie semble « involontaire ».Toutefois, ces erreurs ont provoqué la mort d'homosexuels et les meurtriers s'en sont tirés "sans peine " ou ont réussi à faire réduire les accusations portées contre eux.

Le "crime motivé par la haine " est un concept social relativement récent. Il s'agit d'une nouvelle façon de définir un problème persistant. Selon Jenness, la loi qui régit ce type de crime est fondée sur l'attribution du statut de victime à des groupes donnés, et non à d'autres. De récentes recherches traitent des acteurs sociaux qui "défendent" les victimes homosexuelles et de la façon dont leur discours peut contribuer à cerner les enjeux et à élaborer des positions de principe.

En dépit de la mise en place de lois qui sanctionnent les crimes motivés par la haine aux États-Unis et de la prolifération des travaux de recherche portant sur le sujet, le débat, au Canada, demeure essentiellement axé sur les dispositions qui gouvernent la détermination de la peine et sur la propagande entourant cette catégorie de crimes. Jusqu'ici, l'utilité des dispositions en vigueur n'a guère été démontrée. De fait, je présente dans ce livre des 
Que certaines personnes éprouvent de la haine envers les homosexuels et les agressent est assez triste en soi, mais ce qui est encore plus troublant, c'est que les pratiques juridiques tendent à excuser ce type de violence et à en minimiser l'importance. centaines d'exemples de meurtres et de voies de fait qui se sont produits au Canada après 1995, année où sont entrées en vigueur les dispositions sur la détermination de la peine applicable aux crimes motivés par la haine. Je n'ai trouvé pratiquement aucun rapport indiquant que le juge avait alourdi la peine au motif qu'il s'agissait d'un crime motivé par l'homophobie. Mes recherches semblent donc corroborer la controverse provoquée par Shaffer qui affirmait, en 1995, que les dispositions adoptées n'avaient aucune utilité et qu'il fallait plutôt mettre en place une loi distincte sur les crimes motivés par la haine.

À elles seules, les théories sociologiques et psychologiques ne permettent pas de faire toute la lumière sur la violence homophobe. Pour avoir une idée juste du sujet, il faut examiner de quelle manière l'homophobie influence l'application de la loi. Que certaines personnes éprouvent de la haine envers les homosexuels et les agressent est assez triste en soi, mais ce qui est encore plus troublant, c'est que les pratiques juridiques tendent à excuser ce type de violence et à en minimiser l'importance. Le présent chapitre traite de l'omniprésence de l'homophobie dans le système judiciaire, de l'impossibilité de punir équitablement la violence homophobe dans le système actuel, du mouvement en faveur de l'adoption de lois sanctionnant les crimes motivés par la haine et des recours judiciaires qui s'offrent aux LGBT victimes de violence.

Si des punks entraient dans un restaurant du quartier chinois de Vancouver et se mettaient à saccager les lieux en criant "Chintoks! » et en tabassant les clients, au point de provoquer une émeute sur la rue Pender, il est à parier que l'histoire ferait la une du Vancouver Sun et que la police convoquerait une conférence de presse pour dénoncer ce crime. Pourtant, en 1994, des voyous firent irruption dans un café gai bondé de la rue Davie et se mirent à brutaliser les clients en hurlant "Pédales ", mais l'histoire fut reléguée dans un encadré en page 2 du journal et la police n'y fit même pas allusion à sa conférence de presse hebdomadaire ${ }^{2}$.

Est-ce exagéré de dénoncer le peu d'attention porté par les médias à cette histoire? Je ne le crois pas. L'attaque était un affront envers la communauté gaie. Pis encore, elle s'est conclue de 
"Les cinq hommes pénétrèrent dans le café et se mirent à attaquer les clients en hurlant des slogans anti-gais ${ }^{5} . "$ manière scandaleuse (et sans que les principaux médias en parlent): aucun des agresseurs n'a écopé d'une peine d'emprisonnement. J'étais dans la salle d'audience le jour où on a annoncé qu'une entente avait été conclue entre les parties. Personne ne s'est indigné d'apprendre que la poursuite aboutissait à un non-lieu. Peut-être les gens de Vancouver se sont-ils endurcis face à la violence et à l'inévitable "résolution " des causes. J'ai réussi à déterrer un grand nombre de faits cachés concernant cette histoire après avoir interviewé «Brian ", l'une des victimes.

\section{Fin de soirée au Edge Café}

Le 5 mai 1994, à 2 h 30, Brian rentrait chez lui après être sorti dans une discothèque. Alors qu'il passait devant le Edge Café, un lieu de rencontre gai de la rue Davie, cinq hommes ivres sortirent du restaurant voisin et se mirent à lui crier des insultes homophobes. Ils l'acculèrent au mur latéral du café, où se trouvaient une centaine de clients ${ }^{3}$. Selon Brian, l'un des assaillants pesait $140 \mathrm{~kg}^{4}$. Les cinq hommes pénétrèrent dans le café et se mirent à attaquer les clients en hurlant des slogans anti-gais ${ }^{5}$. "Les voyous lançaient des tasses de café chaud au visage des gens en disant: "Sales pédés, on va tous vous tuer!!" " L'un des copropriétaires du café reçut un coup de tabouret en fer forgé sur la tête, et il fallut 11 points pour suturer la plaie. Les clients se défendirent bravement. Deux d'entre eux, qui avaient réussi à immobiliser deux des agresseurs qui tentaient de s'enfuir, subirent diverses blessures : côtes fêlées et poignet cassé $e^{7}$.

Brian, toutefois, est celui qui s'en est le moins bien tiré: l'un des assaillants, après lui avoir appliqué la prise de l'ours, le projeta violemment contre le trottoir. Il fut grièvement blessé au thorax et eut deux vertèbres broyées. Il mit six mois à se remettre de ses blessures. Malgré la gravité de son état, et le fait qu'il était arrivé à l'hôpital en ambulance, il ne reçut qu'une injection de Démérol et fut invité à rentrer chez lui à pied ou en taxi, à ses frais. Un an et demi plus tard, Brian souffrait de dépression. Il vivait de l'aide sociale, dans une maison de chambres, et devait composer avec $285 \$$ par mois une fois son loyer payé. Il a bien reçu une indemnité de 6500 \$, mais il a consacré la majeure 
« "On a posé à

Brian des questions qui n'auraient pas été posées à d'autres témoins, simplement parce qu'il est homosexuel." " partie de cette somme au remboursement de dettes accumulées pendant sa pénible convalescence. De surcroît, il était atteint de "sinal pilonitis", une infection de l'intestin, et d'une infection de la peau, conséquences de sa médication quotidienne : acide acétylsalicylique, tétracycline, clonazépam, trazodone, pénicilline, cloxacilline, ibuprofene et lithium ${ }^{8}$.

Seulement trois des cinq agresseurs furent mis en accusation. L'enquête préliminaire fut reportée cinq fois et ne commença qu'au bout d'une année 9 . Au cours de l'audience, l'avocat de la défense demanda à Brian s'il était l'ami de l'un des autres témoins. Brian répondit par la négative. L'avocat de la défense poursuivit en décrivant le Edge Café comme "l'ultime endroit où les gais peuvent rencontrer un partenaire sexuel, s'ils ne l'ont pas encore fait " à la sortie des discothèques à $2 \mathrm{~h}$ du matin. Brian se fit demander à trois autres reprises s'il connaissait l'un des témoins, et répondit une fois de plus par la négative. L'avocat lui demanda alors s'il avait déjà couché avec l'un des témoins. Brian haussa le ton : «Je m'objecte à cette question.Vous m'avez demandé quatre fois si je connaissais l'un des témoins et je vous ai répondu que non. Maintenant vous me demandez si j'ai couché avec l'un d'eux. Je vous l'ai dit : "Non!"”

C'est seulement à ce moment que le ministère public souleva son objection. Plus tard, à la question de savoir s'il était normal de demander aux victimes si elles avaient des relations sexuelles avec des passants, le procureur répondit ceci : "On a posé à Brian des questions qui n'auraient pas été posées à d'autres témoins, simplement parce qu'il est homosexuel.»

Dennis Dahl, un avocat deVancouver, croit qu'il y a deux poids, deux mesures : "Lorsque les victimes sont des femmes, le contreinterrogatoire sur leurs antécédents sexuels se fait dans certaines limites. Par contre, si la victime est un homosexuel, la plupart des juges estiment qu'il est pertinent de parler de sa vie sexuelle. Le ministère public a résolument manqué de sensibilité ${ }^{10}$."

Trois mois plus tard, Brian s'adressa aux Services aux victimes pour se plaindre du report continuel des travaux de la cour. Remis trois fois, le procès a finalement eu lieu presque deux ans après 
l'agression. Il semble qu'une douzaine de policiers, croyant que Brian voulait se suicider, aient été dépêchés chez lui avec une ambulance. Brian affirma qu'un policier était entré sauvagement dans son appartement, l'avait injurié, puis poussé contre une table pour lui passer les menottes, l'entravant si étroitement qu'il en avait eu des ecchymoses aux poignets pendant une semaine. Il fut ensuite laissé sans surveillance dans le bureau d'un psychiatre qui était sorti manger. "Si j'étais à ce point une menace pour moimême, déclara Brian par la suite, pourquoi n'a-t-on pas verrouillé la porte de la pièce où je me trouvais? " Au bout d'une heure d'attente, Brian se leva et rentra chez lui à pied ${ }^{11}$.

Deux semaines après le début du procès, alors que dix-sept personnes avaient été appelées à témoigner, la défense et le ministère public négocièrent une entente : un seul des agresseurs plaida coupable de voies de fait ayant causé des lésions corporelles. Il écopa d'une peine avec sursis ${ }^{12}$ et fut forcé de publier une lettre d'excuses à l'une des victimes dans le Angles, un journal de Vancouver s'adressant aux gais qui a cessé de paraître au milieu des années $1990^{13}$.

\section{Travaux de recherche sur l'homosexualité dans le domaine juridique}

Comme le démontre le drame survenu au Edge Café et la consternation qui s'ensuivit, la violence motivée par l'homophobie s'exerce sur trois plans : les blessures physiques infligées aux victimes, l'obligation pour les victimes d'affronter à nouveau les préjugés qui viennent cette fois du système de justice pénale et, enfin, la violence symbolique qui touche toute la communauté lorsque de telles infractions surviennent et que les auteurs semblent s'en tirer impunément.

Certains chercheurs ont tenté de comprendre ce mécanisme mystérieux et parfois préjudiciable. Dans son analyse «des privilèges que retirent les hétérosexuels de la loi », Ryder (1991) 
"...la théorie

queer souligne la

nature mouvante

et contestable des

catégories établies: il

n'y a rien de naturel à

propos des LGBT." souligne qu'il n'est pratiquement pas question des gais et lesbiennes dans la loi, sauf quand vient le temps de présenter les hommes gais comme "des persécuteurs sans scrupules s'en prenant à de faibles victimes ou comme les auteurs de crimes indécents ». Ce mutisme fait de l'hétérosexualité la norme et empêche les homosexuels de s'attendre à la moindre sympathie et de se faire respecter ${ }^{14}$. Selon Robson (1995 : 191), «le vécu des lesbiennes doit se trouver au cœur de la théorie juridique s'appliquant aux lesbiennes ", afin que celles-ci soient la " force centrifuge de l'affaire ${ }^{15}$."

Pour sa part, Stychin $(1995$ : 148) prétend que les étudiants de la common law apprennent habituellement à classer les cas à l'étude dans des catégories de base précises. "Toutefois, la théorie queer souligne la nature mouvante et contestable des catégories établies: il n'y a rien de naturel à propos des $\mathrm{LGBT}^{16}$.» Stychin analyse l'affaire infamante R. c. Brown, qui s'est déroulée en Grande-Bretagne. En 1993, cinq adultes homosexuels consentants qui s'adonnaient à des actes sadomasochistes en privé furent accusés de voies de fait ayant causé des lésions corporelles, même si aucune plainte n'avait été déposée à la police et qu'aucun d'entre eux n'avait subi de blessure permanente. Pendant l'appel, on discuta en cour " du besoin incontrôlable et débridé de violence sexuelle qui domine les homosexuels ${ }^{17}$. " L'un des juges indiqua que «la sodomie, que la loi ne punit plus, est devenue un moyen manifeste de transmission du sida ${ }^{18}$."

Stychin $(1995: 148)$ explique que la Charte canadienne des droits et libertés, " analysée dans une perspective postmoderniste ", trace "certaines lignes de conduite sexuelle " à partir desquelles des identités juridiques, politiques et culturelles peuvent croître et s'approfondir. En revanche, dans la loi américaine, « les distinctions fondées sur l'orientation sexuelle sont en général "rationnelles" et ne donnent pas lieu à un examen juridique approfondi ${ }^{19}$." Backer (1996) signale qu'en 1996, plus de 200 plaintes contestant la constitutionnalité des lois sur la sodomie ont été déposées aux États-Unis. En outre, la sodomie était encore considérée comme un délit dans 23 États ${ }^{20}$ jusqu'en 2003, année où la Cour suprême a finalement abrogé les lois interdisant la sodomie ${ }^{21}$. On s'attendait à un mouvement en ce sens en 1986, alors que la Cour 
" ...au Canada, il est en réalité illégal pour des personnes de moins de 18 ans d'avoir des relations sexuelles anales. Par contre, l'âge de consentement à une relation sexuelle au cours de laquelle il $y$ a pénétration du pénis dans le vagin est de 14 ans dans la plupart des circonstances " suprême était saisie de l'affaire Bowers (1986) ${ }^{22}$, une contestation infructueuse des lois américaines sur la sodomie fondée sur la cause type transparente de deux hommes adultes consentants ayant eu une relation sexuelle en privé. Au lieu du dénouement attendu, la Cour suprême a dû composer avec "des images de prédateurs sexuels, de pédophiles, de prostitués et de pervers ${ }^{23}$ " (Backer, 1996 : 592).

En 2003, les militants gais canadiens célébraient le droit au mariage qui venait de leur être reconnu. Cependant, ils étaient nombreux à ignorer qu'un grand nombre de lois canadiennes réglementent les relations homosexuelles. Comme le signale Kinsman (2003 : 13), la célèbre phrase de Pierre Elliott Trudeau ("L'État n'a pas sa place dans la chambre à coucher des Canadiens.") a été " largement interprétée à tort comme la légalisation des relations homosexuelles ». En fait, les réformes de 1969 ont simplement permis à deux adultes consentants de plus de 21 ans d'avoir des rapports sexuels en privé. Dans les faits, la législation a pris dès lors plus directement pour cibles les relations homosexuelles, grâce à certaines des lois décrites par Kinsman (2003) et Cossman (2003) :

- Relations sexuelles anales (article 159 du Code criminel) : au Canada, il est en réalité illégal pour des personnes de moins de 18 ans d'avoir des relations sexuelles anales. Par contre, l'âge de consentement à une relation sexuelle au cours de laquelle il y a pénétration du pénis dans le vagin est de 14 ans dans la plupart des circonstances ${ }^{24}$. De plus, la loi permet à trois personnes ou plus d'avoir des relations sexuelles en groupe, «pourvu qu'il n'y ait pas pénétration anale.» Cette discrimination dans la loi a été contestée en Ontario, mais « la loi existe toujours et elle demeure exécutoire dans les autres provinces.»

- Actions indécentes (article 173 du Code criminel) : ces actions ne sont pas décrites, cependant les tribunaux se sont entendus pour fixer un "seuil de tolérance social». Cossman (2003 : 15) explique que cet article vise divers actes sexuels, comme les relations sexuelles buccogénitales, la pénétration anale, la masturbation, les danses-contact, l'exhibitionnisme et les contacts sexuels. Ces actes ne sont généralement pas 
réglementés lorsqu'ils se déroulent en privé, mais qu'en est-il lorsqu'ils ont lieu dans des établissements de danse érotique? Il y a plus d'ambiguité dans cette zone grise : les propriétaires et les exécutants « risquent de se faire accuser d'actions indécentes ou de représentation théâtrale immorale ", et les propriétaires de saunas peuvent être mis en accusation « si des indices laissent croire que les clients ont des relations sexuelles à l'extérieur de cabines fermées à clé.»

- Les propriétaires de ces établissements et saunas (où la police juge que des actions indécentes ont lieu régulièrement) peuvent être accusés de tenir une maison de débauche (articles 197 et $210 \mathrm{du}$ Code criminel). Pour leur part, les clients peuvent être accusés de se trouver dans une maison de débauche ${ }^{25}$.

Certains hétérosexuels croient qu'il y a deux poids, deux mesures; ils ignorent peut-être toutes les contraintes que la loi a imposées aux homosexuels. À la fin des années 90, des agents d'infiltration de Montréal se rendirent dans un club échangiste, munis d'une caméra cachée, et y arrêtèrent des hétérosexuels. Après avoir reçu sa sentence, l'un des propriétaires du club s'est plaint : « Les gais font ce qu'ils veulent. Et les hétéros, ils ne peuvent pas faire ce qu'ils veulent? C'est une vraie farce ${ }^{26}$.»

\section{Pratiques homophobes légales au Canada}

En cour, les affaires mettant en cause des homosexuels peuvent froisser les gens. À Milton, en Ontario, des candidats jurés eurent à répondre à des questions sur l'homosexualité avant un procès. Environ $20 \%$ des personnes interrogées indiquèrent qu'elles ne pourraient pas être objectives dans une telle affaire ${ }^{27}$. Un sondage réalisé aux États-Unis auprès de 1012 candidats jurés révéla que " les gais et lesbiennes qui sont parties à un procès ont trois fois moins de chances de bénéficier de l'objectivité du jury que les personnes blanches, noires, hispaniques ou asiatiques ${ }^{28}$. " Pendant une affaire jugée devant un tribunal civil en Californie, les avocats de l'intimé, un homosexuel, ont simulé un procès dans lequel 
"...il arrive parfois que "le juge jette le blâme sur la victime gaie, avançant qu'il est "naturel" qu'une personne "normale" réagisse agressivement face à l'homosexualité." le jury a été formé selon les caractéristiques démographiques du bassin de personnes appelées à faire partie du véritable jury. L'homophobie fortement ancrée des faux jurés était latente. Ils ont donné tort au défendeur, tout comme l'ont fait plus tard les véritables jurés ${ }^{29}$.

Dans un procès qui eut lieu en 1993 à Ottawa, le jury, composé d'un homme et de onze femmes, acquitta un homme accusé du meurtre de Benoît Villeneuve, un prostitué ${ }^{30}$. Un journaliste du quotidien The Ottawa Citizen déclara ensuite que le procès « avait permis une brève intrusion dans le monde sordide de l'itinérance, de la drogue et de la prostitution gaie ${ }^{31}$. " Le corps de Villeneuve fut retrouvé quatre mois après son décès, enterré sous sa maison dans un coffre ${ }^{32}$. L'avocat de la défense, fier de sa victoire, affirma qu'il avait tenté d'obtenir un jury formé de femmes uniquement : "Ça n'a rien de scientifique, a-t-il indiqué, mais comme il était question d'homosexualité dans cette affaire, il était plus probable qu'un jury composé de femmes soit moins homophobe que si des hommes en faisaient aussi partie ${ }^{33}$."

Au Canada, la discrimination contre les queers s'exerce de façon subtile en cour. Casswell affirme qu'il fut décidé, il y a près de 20 ans, que l'homosexualité de l'accusé ne devait être soulevée que si elle avait un lien suffisamment pertinent avec les circonstances des allégations ${ }^{34}$. Cependant, les avocats ont trouvé d'autres moyens de catégoriser les défendeurs pendant l'interrogatoire : Avez-vous déjà été marié? Vivez-vous avec un homme? Trouvez-vous que ce garçon est beau ${ }^{35}$ ?

Il est reconnu que les juges et les procureurs canadiens minimisent l'influence de l'homophobie dans certains crimes motivés par la haine. Comme l'indique MacDougall (2000 :259260), il arrive parfois que «le juge jette le blâme sur la victime gaie, avançant qu'il est "naturel" qu'une personne "normale" réagisse agressivement face à l'homosexualitée ${ }^{36}$." L'histoire suivante est un cas classique où le tort est attribué à la victime. En 1997, à Vancouver, "Sister C " (alias M. Johnson), une "folle " bien connue qui assistait aux célébrations de la Fierté gaie vêtue d'un costume de bonne sœur, fut agressée dans la rue Denman. Un groupe d'hommes gais immobilisèrent le suspect, " M. Jolicœur ", 
jusqu'à l'arrivée de la police. Au procès, le juge rendit une ordonnance de non-lieu, en faisant remarquer ce qui suit :

«M.Johnson a peut-être mal compris l'intention de M.Jolicœur lorsque celui-ci a formulé des commentaires homophobes. [...] M. Jolicœur exprimait simplement sa frustration. [...] Il est clair que M.Jolicœur a été choqué par le costume de M.Johnson. [...] Ainsi, sans le vouloir, [...] M. Johnson a mis M. Jolicœur en colère, et probablement beaucoup d'autres personnes aussi. [...] Peut-être M. Johnson était-il insensible aux penchants sexuels d'autrui, et M. Jolicœur très susceptible sur ce point ${ }^{37}$.»

L'indulgence des tribunaux prend parfois d'autres formes. Dans l'exemple suivant, un adolescent ayant poignardé trois hommes à Ottawa fut accusé de tentative de meurtre. Le juge décida de ne pas renvoyer le jeune devant un tribunal pour adultes, puisqu'il provenait d'une bonne famille - même si, comme le signale MacDougall, « le jeune avait déjà assisté et participé aux activités de gangs de rue, par exemple [...] "en brutalisant des gais avant de les voler". [...] Le juge n'a pas pris en considération l'homophobie profondément ancrée du jeune homme ${ }^{38}$."

À Toronto, Hugh Conroy, un homme séropositif, rentra chez lui un soir en compagnie de Robert Moyer, qu'il avait rencontré dans un pub. Moyer tabassa Conroy et le dévalisa. D'après la sœur de Conroy, celui-ci «n'est jamais parvenu à s'en remettre, et sa santé s'est détériorée rapidement ». Conroy mourut un an après l'attaque. Le représentant du ministère public fit part de son étonnement: "Nous ne savions pas qu'il ne pourrait pas assister au procès, nous n'avons donc pas pu l'interroger comme nous l'aurions voulu. » Comme personne ne pouvait contredire la version de Moyer, le ministère public donna son accord à une peine de 60 jours d'emprisonnement, à purger pendant les week-ends ${ }^{39}$.

Dans un autre ordre d'idées, si un homme qui bat sa conjointe était condamné à travailler dans un refuge pour femmes battues, des voix s'élèveraient à la grandeur du pays. Pourtant, à Saskatoon, un juge a infligé une sentence de 30 heures de travaux communautaires à purger au centre local pour les gais et 
lesbiennes à un homme qui avait frappé un autre homme pendant qu'ils dansaient dans une boîte de nuit gaie et qui s'est ensuite défendu en disant avoir été la cible d'attouchements de la part de la victime ${ }^{40}$. J'ai interviewé une militante qui voyait la chose avec philosophie. En effet, selon elle, plutôt que de créer des lieux protégés contre les agresseurs homophobes, mieux vaut exposer les tabasseurs à la communauté gaie pour les amener à changer d'attitude à l'égard des homosexuels.

La condamnation à des travaux communautaires fut également envisagée lorsqu'un étudiant de Ryerson agressa un homme homosexuel dans la rue Church, en 1991. Dans son jugement de 39 pages, le juge Harris compare les auteurs de violence homophobe aux nazis et aux membres du Ku Klux Klan. Le juge demanda à l'une des victimes de donner son opinion sur la sentence à infliger à son assaillant. Celle-ci suggéra de condamner l'agresseur à effectuer des travaux au Centre communautaire 519 de la rue Church, afin "qu'il soit forcé de voir que les gais et les lesbiennes ne sont pas les odieux personnages qu'il croyait. " Cependant, l'option des travaux communautaires au Centre a été évincée plus tard ${ }^{41}$.

Un autre militant du Centre 519 abonde dans le même sens. À son avis, l'emprisonnement «n'apprend pas aux gens à ne pas nourrir de sentiments haineux. Il faudrait instaurer un système de justice réparatrice novateur pour punir les auteurs d'actes de violence motivés par l'homophobie ${ }^{42}$. » À Port Dover, en Ontario, un assaillant dut payer une amende de $1000 \$$ à un organisme œuvrant auprès des familles et des amis de gais et de lesbiennes ${ }^{43}$. Dans un autre cas, un agresseur de Toronto se vit interdire de vivre dans cette ville ${ }^{44}$. Toutefois, la justice réparatrice s'avère parfois inefficace.

En 1996, un homme gai de Toronto fut assailli et dut recevoir seize points de suture. Le juge qualifia l'agression de « geste vicieux et lâche " à caractère homophobe. Néanmoins, il restait d'avis que les deux assaillants "ne cherchaient pas à s'en prendre aux gais." Les deux accusés se virent infliger une sentence "novatrice " et n'écopèrent d'aucune peine d'emprisonnement : en plus de payer une amende de plus de $1000 \$$, chacun des deux assaillants 
dut rédiger une dissertation. L'un d'eux dut écrire sur la vie d'un «homosexuel célèbre " et l'autre sur "la souffrance d'un homosexuel persécuté en raison de son orientation sexuelle ${ }^{45}$."

Un matin de septembre 1993, les résidents de Winnipeg s'éveillèrent atterrés d'apprendre que trois actes de violence contre des gais avaient eu lieu pendant la nuit. L'un des accusés provoqua la colère du juge, qui déclara : "C'est ainsi qu'est née l'Allemagne nazie ${ }^{46}$. " L'assaillant fut condamné à deux ans d'emprisonnement. Cependant, le juge Huband fit passer la sentence à sept mois, lors de l'appel, expliquant sa décision par le fait que l'accusé était désolé et qu'il s'était dissocié de ses comparses. MacDougall (2000:174) souligne : «Il s'agit du phénomène de la personne qui change soudainement d'opinion sur les homosexuels, juste avant que la sentence soit rendue ${ }^{47}$.»

\section{Critiques formulées par les gais sur le système de justice pénale}

"..." et si nous

avions pu constater que l'agresseur avait attaqué un autre gai récemment, alors nous aurions pu le garder en détention." "
Dans plusieurs cas, l'indulgence apparente cache en réalité l'incapacité du système de punir la violence exercée à l'endroit des homosexuels. Par exemple, après l'agression d'un homme gai à Vancouver, l'ami de la victime déclara ce qui suit : "La police m'a pratiquement dit de fermer ma gueule. [...] On m'a fait comprendre que quelqu'un pouvait pourchasser un gai sur la rue en lui criant "Sale pédé!" mais que, pour autant que cette personne ne le touche pas ou ne lui dise pas “Je vais te tabasser!", elle n'était coupable d'aucun crime ${ }^{48}$. " La police prit les empreintes digitales du suspect et le relâcha. Toutefois, le suspect ne se présenta pas en cour au moment voulu ${ }^{49}$. Lorsqu'on demanda au représentant du ministère public pourquoi l'assaillant avait été relâché, il répondit : "On relâche des gens ayant un dossier criminel de huit pages tous les jours ${ }^{50}$. "Un agent ajouta que s'il existait une loi régissant les crimes motivés par la haine, " et si nous avions pu constater que l'agresseur avait attaqué un autre gai récemment, alors nous aurions pu le garder en détention ${ }^{51}$.» 
"Quand des

pédophiles sont relâchés, la police se donne beaucoup de peine pour en informer les membres d'une communauté.

\section{Devrait-on}

faire de même auprès de la communauté gaie lorsqu'un agresseur condamné pour violence envers les homosexuels est remis en liberté? Don Gunn fut condamné pour homicide involontaire, à la suite du meurtre d'un homme gai en 1986.»
En 1994, un acte de violence contre des gais ayant fait grand bruit à Toronto s'est conclu par l'acquittement des assaillants. Ross Mulhearon et son conjoint Steve se trouvaient à l'extérieur d'un café Second Cup quand six jeunes hommes dans une camionnette s'arrêtèrent devant eux. Ils étaient suivis d'autres amis qui se trouvaient dans une voiture. Mulhearon affirma que l'un des passagers de la camionnette s'était mis à crier "Sales pédés!» et avait lancé une bouteille de bière sur la chaussée. Mulhearon lui demanda alors : "C'est quoi ton problème? " À ce moment, quatre hommes sortirent du véhicule et attaquèrent le couple. Un passant qui tentait de défendre les victimes reçut une bouteille de bière sur la tête, ce qui lui valut vingt points de suture. Une semaine après l'attaque, 250 personnes se réunirent devant le Second Cup et défilèrent dans la rue Yonge pour manifester leur indignation $^{52}$.

Pendant le procès, l'un des accusés avança que Mulhearon avait craché sur la camionnette et avait ainsi provoqué la bataille. De nombreuses versions de l'histoire furent présentées, si bien que l'accusé fut acquitté - ce qui provoqua un tollé dans la salle d'audience ${ }^{53}$. Mulhearon était indigné : « Ils riaient et prenaient du bon temps; pour eux ce n'était rien. " À la suite de cette histoire, Mulhearon écrivit une pièce de thêâtre, qui a été décrite comme " la revanche fantaisiste d'une victime d'actes de violence motivés par l'homophobie, [...] dans laquelle l'agresseur se fait kidnapper et est entravé sur une chaise. " Il proposa de monter la pièce pour faire une levée de fonds au profit du Centre communautaire 519 de la rue Church, mais l'équipe du Centre hésita à appuyer la trame de la pièce et décida de ne pas participer au projet ${ }^{54}$.

Quand des pédophiles sont relâchés, la police se donne beaucoup de peine pour en informer les membres d'une communauté. Devrait-on faire de même auprès de la communauté gaie lorsqu'un agresseur condamné pour violence envers les homosexuels est remis en liberté? Don Gunn fut condamné pour homicide involontaire, à la suite du meurtre d'un homme gai en 1986. En 1993, à Hamilton, il agressa un autre homosexuel dans un commerce de beignets. Toujours à Hamilton, un autre agresseur, relâché en 1998, "aborda un homme de 72 ans et lui 
vola ses biens. L'assaillant, Smith, avait déjà été trouvé coupable de vol à sept reprises, et certaines de ces infractions avaient été commises lors d'agressions d'homosexuels ${ }^{55}$. » En 2004, la police de Toronto fit paraitre un avis pour avertir la communauté qu'un homme de 27 ans avait disparu d'un foyer de transition de Toronto. L'homme était décrit comme un prédateur sexuel de la région de Church-Wellesley ${ }^{56}$.

Dans une autre affaire, un homme ayant à son dossier 50 condamnations se servait des petites annonces pour rencontrer ses victimes, des hommes gais. Après avoir emménagé avec son nouveau conjoint, il lui dérobait ses biens. Il mit un couteau sur la gorge de l'une de ses victimes, un homme d'affaires de Toronto, pour obtenir de l'argent. Après son arrestation, l'agresseur se vanta de son geste auprès de la police. Le juge déclara : «Il est fier de ce qu'il a fait et justifie ses activités criminelles en disant [...] que [la victime est] "juste une pédale" [...]. On ne peut tolérer ce genre de mentalité ${ }^{57}$.» Toutefois, quelles mesures le système de justice pénale a-t-il prises pour empêcher que de tels actes de violence se reproduisent?

\section{Des tueurs qui s'en tirent facilement}

"Dans certains procès, il arrive que des complications procédurales survenant pendant l'enquête ou en salle d'audience permettent aux tueurs d'être acquittés ou de plaider coupable à une accusation réduite. »
Dans certains procès, il arrive que des complications procédurales survenant pendant l'enquête ou en salle d'audience permettent aux tueurs d'être acquittés ou de plaider coupable à une accusation réduite. En 1990, James Gee fut accusé du meurtre de Gerald May, le responsable de l'immeuble où il vivait, dans une banlieue de Vancouver. Gee déclara que May l'avait dragué.Toutefois, la police ayant violé les droits de Gee, elle dut surseoir aux accusations. La petite amie de Gee confia à un agent d'infiltration qu'elle avait aidé Gee à effacer les indices qu'il avait laissés après avoir battu, poignardé et volé la victime ${ }^{58}$.

Un tueur d'Ottawa réussit lui aussi à se libérer d'une accusation de meurtre. Le tout commença dans un bar, alors que Garth Balderston faisait la connaissance d'Ian Anderson. Par la suite, les 
deux hommes seraient allés faire une promenade dans un parc, où Balderston fut battu à mort. Lorsque le corps de la victime fut retrouvé, la bague qu'il portait au doigt avait disparu. Des amis d'Anderson affirmèrent que celui-ci se vantait d'avoir battu un homme gai dans un parc et qu'il leur avait montré une bague volée $^{59}$. Pendant la procédure judiciaire, Anderson embaucha six avocats qu'il congédia l'un après l'autre. L'un d'eux indiqua au juge que l'accusé reconnaissait avoir provoqué la mort de la victime; toutefois, cette déclaration (qui n'a pas été faite devant jury) a été radiée du dossier. Anderson fut condamné pour homicide involontaire ${ }^{60}$.

Dans une autre affaire, une erreur dans la procédure pourrait avoir permis à un homme de 23 ans de se tirer "sans peine " d'une accusation de meurtre pour un crime commis dans un restaurant de Calgary. En 1994, Robert Carolan, complètement ivre, se rendit à la salle de bain du restaurant. Il déclara qu'un homme de 53 ans s'était livré sur lui à des attouchements. La bande sonore de l'enregistrement de la caméra située à l'entrée du restaurant a permis de constater que la victime avait été frappée à 32 reprises. La victime était dans le coma. Lorsque Carolan fut arrêté plus tard dans la rue, il y avait du sang sur ses bottes ${ }^{61}$. Le ministère public tenta de présenter la vidéo comme élément de preuve, mais le juge refusa, expliquant « qu'il était totalement inacceptable de dénaturer les faits et d'influencer les membres de la cour de cette façon ».J'ai lu dans le sommaire du cas que la victime « est décédée plus tard d'un arrêt respiratoire ${ }^{62}$."

J'ai donc appelé le procureur pour savoir si la victime était réellement décédée. On m’a assuré qu'il était impossible que la victime soit morte, car dans le cas contraire le ministère public aurait porté une accusation d'homicide involontaire ou de meurtre. J'ai ensuite téléphoné à la police de Calgary pour savoir si un agent était chargé de vérifier si les victimes blessées au cours d'un incident étaient décédées des suites de leurs blessures. Le chef adjoint à qui j'ai parlé m'a répondu que personne en particulier ne s'en occupait. Cela signifie donc que certains assaillants (et pas seulement des tabasseurs d'homosexuels) peuvent s'en tirer avec une accusation de voies de fait graves, même si la victime 
meurt des suites de l'agression ${ }^{63}$. Ironie du sort, la peine de huit ans d'emprisonnement infligée à Carolan pour voies de fait graves était probablement plus sévère que la peine qu'il aurait eu à purger pour homicide involontaire.

Dans une autre affaire, le décès de Mario Desrosiers a fort bien pu être la conséquence d'une série d'erreurs administratives. La victime rencontra Gregory Hanson à Montréal, à l'été 1994. "Ils étaient toujours ensemble ", raconta un détective après coup ${ }^{64}$. Il semble que la police ignorait que Hanson était recherché pour des crimes violents commis en Californie et en Colombie-Britannique. Par ailleurs, un rapport indique que Hanson « avait conçu un plan audacieux pour s'évader d'une prison de Colombie-Britannique en se cachant sur le toit d'un camion à ordures " en mai $1994^{65}$. Deux mois après le meurtre de Desrosiers, Hanson fut arrêté à Montréal pour voies de fait contre une policière et possession de l'arme qu'il avait utilisée pour commettre un vol. Les policiers de Montréal ne s'étant apparemment pas aperçus qu'ils avaient affaire à un fugitif, ils relâchèrent Hanson après lui avoir infligé une amende de 500 \$ et lui avoir ordonné de se présenter en cour le 23 août 1994. À cette date, Hanson avait fait la connaissance de Desrosiers et l'avait déjà assassiné ${ }^{66}$.

Le crime s'est produit alors que les deux hommes se trouvaient à Gatineau, où il semble qu'ils planifiaient un vol à main armée. Hanson, craignant que Desrosiers rapporte le plan à la police, l'étrangla à l'aide d'une ceinture ${ }^{67}$. Hanson fut arrêté à l'aéroport d'Ottawa le 18 août. Il avait en sa possession les cartes d'identité de Desrosiers et un aller simple pour les îles Fiji. Ce n'est que deux mois après le début de la peine d'incarcération que purgeait Hanson que la police fit le lien entre ce dernier et Desrosiers, les amis de Desrosiers ayant mis des semaines à signaler la disparition de leur copain. Le corps de la victime fut finalement retrouvé des mois plus tard, enterré derrière le poste de police de Hull ${ }^{68}$. Hanson plaida coupable de meurtre au second degré. La sentence prévoyait une possibilité de libération conditionnelle au bout de 13 ans d'emprisonnement ${ }^{69}$. 


\section{Tueurs en liberté conditionnelle}

De nombreux homosexuels ont été assassinés par des agresseurs qui se sont retrouvés dans la communauté gaie après être sortis de prison. En 1992, Terry Fitzsimmons épousa une femme de Kingston alors qu'il se trouvait toujours incarcéré pour le meurtre d'un autre prisonnier.

À peine quelques jours après sa remise en liberté, il avait déjà emménagé avec une autre femme et volé 10000 \$ dans une bijouterie. Pour se cacher, il se mêla à la communauté gaie de Toronto. Il rencontra Don Hebert, « un agent de voyages qui se livrait à la prostitution de temps à autre ". Hebert s'éprit de Fitzsimmons. En 1993, les deux hommes s'embarquèrent dans une odyssée criminelle de six jours qui les mena dans trois villes. Fitzsimmons, âgé de 29 ans, tua trois hommes, dont deux étaient gais.

Hebert, qui était séropositif, s'imaginait que Fitzsimmons et lui étaient les "tout premiers Bonnie et Clyde gais ", même si Fitzsimmons niait être homosexuel. Les deux hommes étaient cocainnomanes, et ils cambriolèrent la même banque de Toronto à deux reprises pour se procurer de la drogue. Les deux malfaiteurs firent la connaissance de Norman Rasky, un dentiste de 62 ans, consommateur de $\operatorname{crack}^{70}$. Ce dernier emménagea avec eux ${ }^{71}$ après s'être fait mettre à la porte de son logement pour défaut de paiement ${ }^{72}$. Un jour que Fitzsimmons était sous l'effet de la drogue, il poignarda et frappa Rasky à plusieurs reprises, tandis que Hebert assistait à la scène. Les deux hommes laissèrent le corps de la victime dans le sous-sol de l'édifice et s'enfuirent à Montréal, où ils dépensèrent tout leur argent. Après avoir tué un chauffeur de taxi, ils prirent la route en direction d'Ottawa ${ }^{73}$.

Le corps de Rasky finit par être retrouvé à Toronto. Un détective de la section des homicides persistait à dire que le meurtre de Rasky "n'avait aucun lien avec son orientation sexuelle et que le crime n'était pas un acte de violence motivé par l'homophobie ${ }^{74}$. " La police de Toronto lança un appel à l'aide 
dans la communauté gaie afin de retrouver les deux hommes: «M. Fitzsimmons fait $1,70 \mathrm{~m}$ et pèse $69 \mathrm{~kg}$. Il a de nombreux tatouages sur les deux bras et la poitrine. M. Hebert mesure 1,78 $\mathrm{m}$. Il a une moustache foncée, une cicatrice de $7 \mathrm{~cm}$ sur le côté droit du cou et un tatouage représentant le drapeau du Canada sur l'épaule droite. La police croit que les deux hommes se seraient rasés les cheveux pour changer d'apparence ${ }^{75}$."

Le soir de leur arrestation, les deux hommes furent aperçus dans un bar d'Ottawa vêtus du même chandail des Blue Jays, l'équipe de base-ball de Toronto ${ }^{76}$. Ils terminèrent la soirée dans un restaurant vide de la rue Bank. Selon la déclaration de Fitzsimmons, Hebert lui aurait confié qu'il ne supportait pas l'idée d'être emprisonné pour les meurtres qu'il avait commis. Fitzsimmons l'étrangla alors avec un t-shirt, "s'injecta dans les veines deux fioles du sang contaminé de son ami " et "enfonça un couteau de boucherie dans le cœur de Hebert ». Fitzsimmons téléphona ensuite à la centrale d'urgence, au 911, puis il prit une dose de cocaïne et se rendit à un poste de police. Il déclara au réceptionniste : «Il faut que ça cesse. Je n'en peux plus de tuer des gens. »

Fitzsimmons raconta que Hebert et lui avaient fait un pacte de suicide. Il ne voulait pas que l'état de santé de Hebert «se détériore à cause du sida. Ils avaient créé une sorte de fraternité de condamnés ${ }^{77}$. Fitzsimmons indiqua qu'il voulait que la mort vienne à lui par le sang de Hebert, qu'il décrivit avec émotion comme "le meilleur ami qu'il ait jamais $e^{78}$.» Néanmoins, il voulait rectifier les "erreurs au sujet de sa relation avec Hebert, que les médias avaient faussement dépeinte. » Il expliqua : "Cela influence la façon dont les autres prisonniers te traitent ${ }^{79}$. " $\mathrm{A}$ l'annonce de la sentence, l'avocat de Fitzsimmons soutint que la mort de Hebert était le résultat d'une " combustion spontanée, provoquée par la consommation de drogue, les années passées en prison sans contact avec le monde extérieur et l'attitude hostile de son agent de libération conditionnelle." Le juge affirma que le meurtrier était "dépravé " et le condamna à 16 ans d'emprisonnement avant toute possibilité de libération conditionnelle ${ }^{80}$. Fitzsimmons se suicida en $1995^{81}$. 
«McGray assomma Assaly à l'aide d'une lampe et le poignarda à 16 reprises, puis il partit à la recherche de sa victime suivante."
L'histoire d'un autre tueur en libération conditionnelle, Michael McGray, a fait les manchettes. En 2000, McGray se déclara le pire tueur en série de l'histoire du Canada. Détenu dans une prison à sécurité minimale du Québec en 1991, il eut droit à un congé de trois jours pendant la période de Pâques. Il passa le week-end à Montréal, où il commit un homicide. McGray raconta qu'il était l'un des "préférés » de son père quand venait le temps de distribuer les coups et qu'il avait été agressé sexuellement par les gardiens d'un établissement pour garçons de la NouvelleÉcosse ${ }^{82}$. De plus, il expliqua qu'il était « très difficile d'étrangler quelqu'un et qu'il aimait commettre des meurtres lorsqu'il ne se trouvait pas sous l'effet de l'alcool ou de la drogue, "parce qu'il pouvait ainsi savourer son geste" ». Il déclara qu'il n'était pas gai, ajoutant : "C'est incroyable à quel point ils t'invitent facilement chez eux, même si tu es un parfait inconnu ${ }^{83}$."

La police de Saint-Jean au Nouveau-Brunswick découvrit que McGray était celui qui avait poignardé James Lloyd Beyea, un homme gai surnommé "Fluff», en $1986^{84}$. Par ailleurs, McGray avait plaidé coupable à une accusation de meurtre au premier degré en 1991. Ses victimes étaient Robert Assaly, un homme de 59 ans à la retraite qui avait été professeur dans la communauté libano-chrétienne, et Gaëtan Éthier, un vendeur sans emploi de 45 ans. Selon la version des événements donnée par McGray, il avait rencontré Assaly dans le "Village gai », aurait pris quelques verres avec lui et l'aurait accompagné à son condo. McGray assomma Assaly à l'aide d'une lampe et le poignarda à 16 reprises, puis il partit à la recherche de sa victime suivante. Le corps d'Assaly fut découvert par son frère une semaine plus tard.

De retour dans le Village, McGray fit ensuite la connaissance d'Éthier, qui lui aussi invita l'assassin chez lui. McGray raconta qu'une fois Éthier endormi, il lui avait asséné un coup à la tête avec une bouteille de bière et l'avait poignardé. McGray quitta ensuite Montréal. La police l'arrêta trois semaines plus tard, parce qu'il avait enfreint d'autres conditions de sa mise en liberté sous condition, et non pas parce qu'il avait commis des meurtres. Il fallut neuf ans à la police pour faire le lien entre McGray et les meurtres commis à Montréal. Le frère de Robert Assaly insista 
pour dire que son frère n'était pas gai, qu'il travaillait simplement dans un bar duVillage gai ${ }^{85}$. Il est facile de comprendre pourquoi le frère d'Assaly croyait que celui-ci était hétérosexuel. Je me suis entretenu avec un couple d'amis hétérosexuels qui étaient proches de Robert; ils avaient enseigné avec lui et avaient fait de longs voyages à l'étranger en sa compagnie. Ils ont affirmé que rien n'avait pu leur faire croire qu'Assaly était homosexuel et que, si c'était vraiment le cas, ils étaient attristés par le fait qu'Assaly n'avait pas osé leur avouer son orientation sexuelle.

\section{La construction sociale du crime motivé par la haine}

«Mason (2001:

256) définit le crime motivé par la haine comme "l'expression physique, à l'endroit d'une personne, d'un sentiment de haine fondé, en tout ou en partie, sur le fait que l'on reconnaît chez elle les caractéristiques d'un groupe précis. »"
Jenness (1999 : 549) signale que "les réformes judiciaires sont le principal moyen de contrer la violence motivée par la haine ${ }^{86}$." J'ai démontré jusqu'ici que la manière dont certaines lois sont appliquées (et le fait que d'autres lois ne sont pas appliquées ou le sont avec réserve) crée un environnement propice à la violence qui s'exerce contre les gais. Dans la présente section, je traiterai du mouvement qui est à l'origine du concept de "crime motivé par la haine " et des lois qui régissent ce type de crimes.

Mason (2001 : 256) définit le crime motivé par la haine comme "l'expression physique, à l'endroit d'une personne, d'un sentiment de haine fondé, en tout ou en partie, sur le fait que l'on reconnait chez elle les caractéristiques d'un groupe précis. " Elle rappelle l'histoire d'une militante qui s'est enfuie alors que des gais se faisaient attaquer, sous prétexte qu'il ne s'agissait pas « de la bonne bataille " pour elle. Peut-être cette militante n'était-elle en fait pas à l'aise de travailler avec des "pédés, des poules et des prostitués ${ }^{87}$."

De son côté, Rosga fait une nouvelle analyse du cas de la "femme brûlée ", un affreux crime motivé par la haine perpétré au Maryland. Rosga s'entretint avec la police pour connaitre la version "médiatisée " de l'histoire ainsi qu'avec l'un des hommes " racistes » et "misogynes » coupables de ces actes de "sauvagerie " et de « lynchage ». L'homme affirma qu'il avait de nombreux amis 
noirs et qu'il ne s'était même pas rendu compte que la victime était noire ni qu'il s'agissait d'une femme. Il prétendit avoir été injustement dépeint comme un "fauteur de troubles haineux " par un juge juif, dont la femme s'était démenée pour faire promulguer par l'État une loi sur les crimes motivés par la haine ${ }^{88}$.

Jacobs et Potter (1998: 46) mettent en doute la prétendue "épidémie " de crimes motivés par la haine qui frapperait l'Amérique et dont " parlent sans cesse les politiciens, les journalistes et les chercheurs." Les groupes de gais et lesbiennes " figurent parmi ceux qui proclament avec le plus d'éclat l'existence d'une telle épidémie ${ }^{89}$." Les auteurs reprochent à Levin et McDevitt d'accorder trop de crédit aux données provenant des groupes de pression ${ }^{90}$ et contestent leur principale théorie selon laquelle il y a de plus en plus de crimes motivés par la haine en raison de " l'affaiblissement de l'économie et de la tension psychosociale qui en découlent. " Jacobs et Potter (1998 :53) dénoncent le manque de preuves empiriques à la base d'une "théorie qui n'a pu mettre le doigt sur le problème ${ }^{91}$."

Jenness (1999) a signé et cosigné plusieurs analyses des lois sur les crimes motivés par la haine et du mouvement qui cherche à endiguer ce genre de crimes aux États-Unis. L'auteure se penche sur les travaux des comités du Congrès réalisés dans les années 80 et 90 , qui ont donné lieu à la promulgation, dans les années 90, de trois lois fédérales sur les crimes motivés par la haine. Elle a analysé les mesures prises par les organismes sociaux pour « influencer la manière dont les décideurs interprètent les enjeux et en traitent afin de conférer aux lois une raison d'être jusqu'à ce qu'elles soient adoptées et bien établies. » Une partie de ce processus revient à élaborer des définitions socialement construites "qui ont pour conséquence que l'on assigne un statut de victime à certaines personnes ou à certains groupes, et pas à d'autres ${ }^{92}$." Jenness (1999: 553) cite les éléments suivants :

- les «intervenants à l'origine de chacun des projets de loi : militants, politiciens, représentants des mouvements sociaux, victimes et survivants d'actes de violence ";

- les "arguments présentés en faveur et à l'encontre " de ces projets de loi; 
- la manière dont la race, la religion, l'ethnicité, l'orientation sexuelle et le sexe sont "caractérisés, définis, invoqués et négociés durant la rédaction des lois fédérales régissant les crimes motivés par la haine ${ }^{93}$."

$\mathrm{Au}$ début, les gens réclamaient le droit au respect de la différence de race, de religion et d'ethnicité. Par la suite, «ce champ juridique a commencé à s'étendre ", élargissant ainsi "le cadre délimitant ce phénomène jugé problématique ». En ce sens, les groupes de gais et lesbiennes ont joué « un rôle prépondérant en luttant en faveur de l'élargissement de la portée de la loi.» S'ils y sont parvenus, c'est en partie parce qu'ils ont comparé la violence motivée par l'homophobie à la violence motivée par la haine qui était déjà reconnue dans une certaine mesure. Pendant ce temps, les partisans du camp adverse dénonçaient la «propagande des militants homosexuels » et se refusaient à admettre que la violence envers les gais n'était pas différente de la violence envers d'autres minorités ${ }^{94}$.

Dans un autre article, Jenness (2001) souligne qu'avant même que ne soit popularisé le concept de crime motivé par la haine, des mouvements plus discrets de défense des droits des minorités avaient déjà réussi à politiser la violence à l'égard de certains groupes minoritaires, bien que dans une moindre mesure. Parallèlement, un nombre croissant de victimes de crimes commençaient à unir leurs voix pour réclamer "que leur soient accordés une aide et un soutien particuliers et que leurs droits, à titre de victimes de crimes, leur soient reconnus. " Aux États-Unis, le contexte était donc propice à la création d'un mouvement de lutte contre les crimes visant les minorités, mouvement qui "donna naissance à l'expression "crime motivé par la haine" et qui en définit les principales caractéristiques. Ce regroupement demanda par ailleurs aux législateurs et aux autres décideurs d'admettre que la violence motivée par la haine constituait un problème social important ${ }^{95}$."

Le Congrès des États-Unis passa trois lois contre les crimes motivés par la haine ${ }^{96}$, qui ne s'appliquent toutefois qu'aux crimes commis sur le territoire et dans les propriétés de compétence fédérale : 
- La Hate Crime Statistics Act (Loi sur les statistiques relatives aux crimes motivés par la haine), adoptée en 1990.

- La Violence Against Women Act (Loi sur la violence envers les femmes), adoptée en 1994. Cette loi a depuis été déclarée inconstitutionnelle. Cependant, dans le cadre de cette loi, plus d'un milliard de dollars ont été investis dans "l'éducation, la mise en place de services d'aide téléphonique pour les victimes de viol, la formation du personnel juridique, les services d'aide aux victimes et les services de police."

- La Hate Crime Sentencing Enhancement Act (Loi sur le renforcement des peines infligées pour les crimes motivés par la haine), adoptée en 1994. Cette loi permet aux juges, dans le cas de huit infractions précises allant du vandalisme au meurtre, d'alourdir les peines, qui «peuvent aller jusqu'à correspondre à la peine infligée pour une infraction dont le niveau de gravité est trois fois plus élevé. " ${ }^{97}$

Dans un livre rédigé en collaboration, Jenness et Grattet (1996) avancent que le crime motivé par la haine est « un problème qui existe depuis toujours, mais qu'on aborde maintenant sous un angle nouveau et qu'on cherche à régler sans délai. » Ainsi, ce qui était auparavant "un crime ordinaire est maintenant un type de crime bien défini, sanctionné plus sévèrement qu'avant. " Cette nouvelle tendance cache en fait le désir "d'envoyer un message symbolique à la société, à savoir que les actes criminels motivés par la haine ne seront pas tolérés ${ }^{98}$."

Dans leur analyse, les auteurs définissent ce type de crime comme « un nouveau domaine dans lequel l'État doit établir des politiques et exercer son pouvoir. » Par conséquent, les auteurs ne se concentrent pas tant sur les crimes comme tels, mais plutôt sur la construction sociale des "schèmes servant à définir et à classifier " ces crimes. La première question que présuppose la détermination de politiques dans un nouveau domaine est la suivante: Quels sont les acteurs qui interviennent, " par exemple, les politiciens, les experts et les représentants d'organismes et de groupes d'intérêts [...] qui ont acquis la crédibilité voulue pour traiter du sujet et faire avancer le dossier? " Une deuxième question se pose : Quels sont «le contexte culturel, les théories, le cadre social et 
"Les auteurs insistent pour préciser que la mise en place de politiques va bien au-delà de la simple élaboration de lois.» les idéologies » qui concourent à la définition du problème et au choix de la solution politique appropriée?

Les auteurs insistent pour préciser que la mise en place de politiques va bien au-delà de la simple élaboration de lois. En effet, les politiques «sont renégociées et redéfinies à différentes étapes." Le processus au bout duquel les nouvelles politiques seront mises en application peut être divisé en quatre phases qui empiètent les unes sur les autres :

- La reconnaissance du problème; on "admet qu'il existe un problème social et qu'il faut le régler, et on nomme le problème. "

- Le choix de la solution; on "détermine quelle est la solution à adopter parmi un éventail de possibilités.»

- L'élaboration des règles; on " étoffe » le contenu des règles.

- L'application concrète; les règles sont établies et appliquées par les organismes compétents.

Ainsi, les auteurs démontrent «comment les mouvements sociaux ont fait de la violence motivée par la haine un problème à traiter, comment les politiciens [...] ont tracé les paramètres relatifs à ce type de crime en instaurant des lois, comment les tribunaux ont élargi la définition du "crime motivé par la haine", comment les responsables du maintien de l'ordre classent ces crimes, mènent les enquêtes requises et entament des procédures pour punir ce comportement criminalisé par la loi ${ }^{99}$."

La mise en place de lois n'est toutefois pas la dernière étape : "Les juges sont ceux qui déterminent en grande partie ce qui constitue un crime motivé par la haine en appliquant les lois à des situations "concrètes". Au fil du temps, [...] les juges établissent précisément ce qui entre dans le champ d'application de la loi. » Les cours d'appel jouent un rôle particulièrement important, puisque les juges de ces tribunaux "peuvent étendre ou complexifier le sens d'une loi, en circonscrire le sens et l'application, en récuser le libellé, valider ou tenir compte d'autres lois, approfondir les motifs [...] et décider s'il convient d'élargir la portée de la loi pour prendre en considération de nouveaux facteurs. " 
Les auteurs ont analysé 36 contestations de la constitutionnalité de la loi sur les crimes motivés par la haine. Ils ont relevé cinq motifs principaux de contestation : manque de précision de la loi; sanction des propos haineux; trop grande portée de la loi; réglementation des propos haineux; inégalité de la protection conférée par la loi ${ }^{100}$.

Jenness (2001) avance dans une autre source que le traitement " égal » de tous les groupes, dans le cadre de la procédure judiciaire, crée une certaine "homogénéité "; la loi ne peut pas accorder de protection à un groupe particulier sans que les autres groupes puissent aussi en bénéficier. C'est la raison pour laquelle les lois sur les crimes motivés par la haine sont exemptes de catégories comme « la violence contre les Noirs " ou " la violence contre les Juifs ", mais comportent plutôt des termes généraux tels que "la race » et « la religion ».Ainsi, un crime motivé par la haine commis contre la race blanche ou l'hétérosexualité est considéré comme aussi grave qu'un crime perpétré à l'endroit d'une personne du simple fait qu'elle est sikhe ou transsexuelle. Cela semble " aller de soi »; cependant, " en classant les personnes [...] dans des catégories englobantes et neutres en apparence ", on oublie les raisons pour lesquelles la loi a été mise en place et on perd de vue la définition de "crime motivé par la haine ".

Puisqu'elles regroupent différents types de crimes sous une seule catégorie, les lois sur les crimes motivés par la haine assurent "l'homogénéité entre les catégories", de sorte que les crimes motivés par la haine des personnes handicapées équivalent aux crimes "motivés par la haine des musulmans ». Jenness (2001) condamne le "silence" auquel ont été réduits les mouvements sociaux qui sont à l'origine des débats. De fait, il s'est produit un " clivage net " entre les « motifs de l'adoption des politiques sanctionnant le crime motivé par la haine, l'élaboration de ces politiques (la loi) et leur mise en vigueur (l'application de la loi) ${ }^{101}$."

Jenness et Grattet (1996) ont constaté, à partir des examens juridiques qu'ils ont analysés, que le "sens profond " du crime motivé par la haine a évolué dans deux directions. La définition juridique est devenue plus nuancée, et les tribunaux « soutiennent qu'il est "plus que jamais dans l'intérêt" des gouvernements de 
réfréner les crimes motivés par la haine ${ }^{102}$.» En outre, les tribunaux ont tenté de préciser dans quelle mesure la haine doit être ce qui a poussé l'agresseur à agir pour que son acte soit considéré comme un crime motivé par la haine. La tendance consiste à considérer le mobile de la haine comme un facteur important plutôt que comme le facteur principal ${ }^{103}$.

Dans quelle proportion les procureurs fondent-ils leurs accusations sur la haine qui a motivé l'agresseur? Les auteurs soulignent qu'il n'existe pas de statistiques sur le sujet, en raison de "la création récente de cette catégorie de crimes ». Néanmoins, pour évaluer les « résultats » d'une loi, on peut calculer le nombre total d'infractions signalées qui ont donné lieu à des poursuites. Selon des recherches préliminaires effectuées en Californie, le taux de judiciarisation des crimes motivés par la haine ne fluctue guère si on le compare à celui de tous les autres types de crimes.

Les auteurs suggèrent une autre façon d'analyser la question : "calculer la proportion des poursuites intentées contre des agresseurs motivés par la haine qui ont abouti à une condamnation. " En Californie, où il est maintenant bien établi que certaines infractions constituent des crimes motivés par la haine, les données préliminaires révèlent « qu'il n'est pas plus difficile d'obtenir une condamnation pour ce type de crime que pour les autres. " Il s'ensuit que « les procureurs de la Californie déposent maintenant plus souvent qu'avant des plaintes pour des crimes motivés par la haine. Le nombre de condamnations et de cas de reconnaissance de la culpabilité des accusés augmente également, ce qui laisse croire que les procureurs hésitent de moins en moins à invoquer les lois sur les crimes motivés par la haine et que les avocats de la défense ont moins tendance à les en empêcher ${ }^{104}$."

\section{Lois sur les crimes motivés par la haine au Canada et à l'étranger}

Comme je l'ai souligné plus haut, aux États-Unis, on tend de plus en plus à criminaliser les infractions motivées par la haine. 
«...aux États-Unis, on tend de plus en plus à criminaliser les infractions motivées par la haine. Toutefois, au Canada, aucune loi ne place ce type d'acte de violence au rang des crimes. "
Toutefois, au Canada, aucune loi ne place ce type d'acte de violence au rang des crimes. En 2003, l'organisme Égale Canada et d'autres groupes de défense des droits des LGBT ont incité la communauté à appuyer le projet déposé par le député gai Svend Robinson à la Chambre des communes, qui va dans ce sens. Dans le débat qu'a soulevé ce projet de loi, certains intervenants ont fait référence, à plusieurs reprises, aux victimes de la violence contre les LGBT, ce qui a donné au public l'impression que la loi proposée portait sur la violence envers ces victimes. En réalité, le projet de loi de M. Robinson (projet de loi émanant des députés portant le numéro C-250) avait pour objet de faire modifier la loi sur la propagande haineuse. Comme l'indique Roberts (1999: 4), "certains auteurs ne font aucune distinction entre le crime motivé par la haine et la propagande haineuse ${ }^{105}$.» Pourtant, il s'agit de deux concepts bien distincts.

Jusqu'en 2004, les articles 318 et 319 du Code criminel interdisaient le génocide et l'incitation à la haine envers des groupes fondée sur la couleur, la race, la religion ou l'origine ethnique; il n'y était pas fait mention de l'orientation sexuelle. Ainsi, en 1995, un résidant de Winnipeg réussit à faire paraitre une brochure dans laquelle il incitait les citoyens à tuer des homosexuels ${ }^{106}$. De plus, en 1997, des internautes reçurent le courriel suivant : "Mort aux homosexuels, comme l'ordonne la Bible! Homosexuels, prenez garde pendant la prochaine semaine de la Fierté gaie!!! " Ce message était signé : "La nouvelle patrouille de tabasseurs de gais de Winnipeg ». La police n'a pu intenter de poursuites dans aucun des deux cas ${ }^{107}$.

En 1999, Svend Robinson demanda à la ministre de la Justice d'ajouter l'orientation sexuelle au nombre des critères définissant les groupes visés aux articles susmentionnés. La ministre répondit qu'elle allait étudier la question. Deux ans plus tard, elle affirma qu'elle n'avait toujours pas terminé l'étude du dossier. Néanmoins, les ministères de la Justice de toutes les provinces ainsi que l'organisme Canadian Association of Police Boards avaient appuyé le changement proposé. Une fois le projet de loi déposé, le député Robinson dénonça le geste de la droite religieuse, qui "avait inondé les bureaux des députés de messages prédisant 
que le changement aurait des conséquences apocalyptiques." Un député de l'Alliance canadienne, un parti de droite, déclara à M. Robinson que «le projet de loi allait à l'encontre de la Bible et du catéchisme catholique ${ }^{108}$. $"$ Le projet de loi reçut la sanction royale le 29 avril $2004^{109}$.

Au terme de ce débat, certains Canadiens croient que le Code criminel contient maintenant une disposition condamnant la violence motivée par la haine. En réalité, il n'en est rien. Seul le sous-alinéa 718(2)a)(i) aborde la question. Cette disposition porte sur la détermination de la peine et donne au juge la possibilité d'alourdir la sentence lorsqu'il peut être prouvé hors de tout doute raisonnable que l'infraction a été motivée par la haine. On n'a que peu discuté du rôle purement symbolique de cette disposition et de son inefficacité.

En analysant les lois sur les crimes motivés par la haine en vigueur dans trois pays, Roberts (1999) a constaté que l'alourdissement des peines prononcées contre les auteurs de tels crimes se faisait selon trois modèles. Le premier consiste à établir clairement " un niveau de gravité élevé ou une peine minimale dans le cas d'une telle infraction " en invoquant les dispositions des lois d'application prévoyant des peines obligatoires. Le deuxième modèle prévoit le recours aux tribunaux d'appel; ce sont donc ces derniers qui fixent la sentence finale. Selon le troisième modèle, celui qui est en place au Canada, la " loi établit les circonstances aggravantes " dont les juges peuvent à leur discrétion tenir compte au moment d'établir la peine ${ }^{110}$. De façon générale, « la solution légale la plus populaire pour sévir contre les auteurs de crimes motivés par la haine consiste [...] à alourdir la peine infligée ${ }^{111}$."

Au Royaume-Uni, la Crime and Disorder Act (Loi sur les crimes et l'inconduite) établit de façon précise ce qui constitue un crime motivé par la haine. Par conséquent, la définition d'une infraction motivée par le racisme, par exemple, a donné lieu à l'apparition de nouvelles catégories de crimes, comme les voies de fait aggravées par le racisme ou le harcèlement aggravé par le racisme. Cette loi du Royaume-Uni fournit également aux tribunaux un facteur "permettant de vérifier s'il y a eu circonstance aggravante ». Il est considéré qu'une infraction 
«Aux États-Unis, ... La loi définit ce type de crime de façon globale; un crime peut être motivé par la haine " en tout" ou "en partie »." est aggravée par le racisme si "au moment de l'infraction, ou immédiatement avant ou après, le contrevenant démontre de l'hostilité envers la victime sur la base de son appartenance (ou de la présomption de son appartenance) à un groupe racial. »

Roberts voit des avantages à cette approche :le terme "aggravé par le racisme" est plus nuancé que le terme "motivé par le racisme". En outre, "le terme "immédiatement" [...] fixe une balise temporelle " qui "dépasse la portée des lois d'autres pays, où les actes de haine doivent avoir eu lieu avant l'infraction et avoir motivé l'attaque ${ }^{112}$.»

Au Royaume-Uni, la même loi prescrit clairement que " toute infraction doit être punie plus sévèrement s'il est établi qu'elle a été motivée par le racisme ». Autrement dit, les juges n'ont pas de pouvoir discrétionnaire. S'il est prouvé hors de tout doute raisonnable que l'infraction est aggravée par le racisme, le juge doit en tenir compte au moment de déterminer la peine et, en plus, il « doit déclarer en cour que de telles circonstances aggravantes existent ". Par ailleurs, cette loi prévoit des peines maximales plus sévères dans les cas de crimes aggravés par le racisme. Ainsi, alors que la peine maximale normalement prononcée pour voies de fait est de six mois d'emprisonnement, si le contrevenant est condamné pour voies de fait motivées par le racisme, il peut devoir servir jusqu'à deux ans d'emprisonnement ${ }^{113}$.

Aux États-Unis, la Hate Crime Statistics Act, adoptée en 1990, avait comme avantage de fixer des lignes directrices claires pour aider le secrétaire à la Justice à déterminer ce qui constituait ou non un crime motivé par la haine. La loi définit ce type de crime de façon globale; un crime peut être motivé par la haine " en tout" ou " en partie ». De surcroit, la loi dresse une liste "d'infractions de prédicat ou d'infractions primaires : homicide involontaire, viol, voies de fait graves, voies de fait simples, intimidation, incendie criminel, destruction de biens, dégâts matériels et vandalisme ${ }^{114}$."

En application de la Hate Crime Sentencing Enhancement Act de 1994, "la peine infligée pour ce type de crime est "alourdie" de façon à correspondre au minimum à la peine infligée pour 
une infraction de niveau trois fois plus élevé.» La "durée considérablement plus longue de la peine d'emprisonnement " est déterminée à l'aide d'une grille dans laquelle sont pris en considération «la gravité du crime et les antécédents criminels du contrevenant ${ }^{115}$.»

En 1999, plus de 40 États américains avaient adopté des moyens légaux pour pénaliser les crimes motivés par la haine. Plusieurs États ont alourdi les peines prévues en établissant une nouvelle classification des crimes motivés par la haine: par exemple, "l'auteur d'une haute trahison de deuxième degré devient passible d'une peine correspondant à un acte délictueux grave. » Certains États considèrent même que la haine « constitue une circonstance aggravante pouvant entraîner l'imposition de la peine de mort plutôt que l'emprisonnement à perpétuité sans possibilité de mise en liberté sous condition ${ }^{116}$.» Ainsi, un meurtrier du Texas fut condamné à la peine de mort (plutôt qu'à l'emprisonnement à perpétuité) après qu'il eut admis avoir tué sa victime en raison de son homosexualité ${ }^{117}$.

\section{Débat en cours au Canada au sujet de la loi sur les crimes motivés par la haine}

"..." qu'il ne servait pas à grand-chose de chercher à éliminer la haine, puisque les causes profondes des préjugés subsistaient et que de nouveaux cas de violation des droits de la personne pouvaient même survenir. » »
En 1995, pendant le débat relatif au projet de loi C-41 (qui a mené à l'adoption des dispositions sur l'alourdissement de la peine de l'article 718), Giese (1995: 7-9) déclara « qu'il ne servait pas à grand-chose de chercher à éliminer la haine, puisque les causes profondes des préjugés subsistaient et que de nouveaux cas de violation des droits de la personne pouvaient même survenir." L'appel aux représailles lancé par la communauté LGBT pendant le procès d'un agresseur homophobe préoccupa l'auteure : « La revanche (et l'imposition d'une peine) constitue une solution rapide et procure un soulagement temporaire face à des problèmes complexes. » Elle poursuit en affirmant que « le gouvernement a su prendre le train en marche et profiter du mouvement de lutte 
contre les crimes motivés par la haine. Il peut ainsi prétendre que les mesures qu'il a prises pour défendre les droits des minorités sont beaucoup plus importantes qu'elles ne le sont en réalité ${ }^{118}$.»

Selon Jeffrey (1998), bien que les médias et les groupes de pression aient dépeint le projet de loi C-41 comme un changement notable, dans les faits, les dispositions " ne réduisent pas l'important pouvoir discrétionnaire des juges, qui décident du poids qu'ils accordent à chacun des facteurs au moment de déterminer la peine. » Quant à savoir si les juges tiennent compte de ces dispositions au moment de prononcer la sentence, il n'est pas possible de le déterminer, " car ils ne sont même pas tenus, à cette étape de la procédure, de citer les nouvelles dispositions ${ }^{119}$."

Il est surprenant de constater le peu d'attention que portent les théoriciens à cette question au Canada. Shaffer (1995) a rédigé un article sur l'inefficacité du projet de loi C-41, alors que la loi venait tout juste d'être édictée. Elle appuie sans réserve la mise en place d'une loi distincte régissant les crimes motivés par la haine, loi qui statuerait que "de tels actes de violence causent une forme précise de préjudice ${ }^{120}$ ", puisque certaines lois criminelles jouent " un rôle normatif ou symbolique pour les citoyens en leur indiquant les comportements désapprouvés socialement ». Par ailleurs, une loi distincte permettrait de recueillir des statistiques plus précises concernant ce type de crimes puisque, à l'heure actuelle, « il arrive que le ministère public et la défense s'entendent pour dissimuler certains détails du mobile du crime au juge lorsqu'ils négocient un plaidoyer ${ }^{121}$."

Shaffer (1995) se demande également si les gouvernements ne se hâtent pas d'adopter des lois sur les crimes motivés par la haine "parce qu'il s'agit pour eux d'un moyen facile de faire valoir qu'ils s'efforcent de régler un problème social. » De fait, la mise en place d'une loi nécessite relativement peu d'investissements et suscite " une grande attention de la part des médias, tout comme le font les procès dans lesquels sont invoquées les dispositions qui régissent ce genre de crime. Le retentissement médiatique permet au gouvernement de se faire du capital politique » d'une manière plus flamboyante que ne le permet le travail de sensibilisation à petite échelle ${ }^{122}$. 
Comme les réformes de la loi criminelle n'entraînent habituellement pas de véritable changement social, Shaffer estime que le pouvoir que conferent les dispositions sur la détermination de la peine est largement « illusoire ». En effet, les juges ne sont pas tenus d'utiliser ces lignes directrices et, même s'ils le font, ils n'ont pas à expliquer dans quelle mesure ils ont alourdi la peine lorsqu'ils prennent la haine en considération comme facteur aggravant ${ }^{123}$. Dans l'ensemble, Shaffer est d'avis qu'il vaudrait mieux adopter au Canada des lois distinctes sur la criminalité motivée par la haine, comme les États-Unis l'ont fait ${ }^{124}$.

Au cours de ma recherche, j'ai analysé plus d'une centaine de cas d'agressions à l'endroit des LGBT qui se sont produits depuis que les dispositions sur la détermination de la peine ont pris force de loi au Canada en 1995. Dans aucun de ces cas, les médias n'ont mentionné que les dispositions sur l'alourdissement de la peine avaient été appliquées.

En comparaison avec les mesures prises aux États-Unis et en Grande-Bretagne, les dispositions sur la détermination de la peine en vigueur au Canada sont plutôt vagues : l'article 718 établit que la peine doit être "adaptée » aux circonstances aggravantes fondées sur la haine.Toutefois, Roberts (1999:19) souligne : «Jusqu'à quel point ces circonstances doivent-elles alourdir la peine imposée? Aucune orientation n'est fournie, et la peine maximale demeure la même : par exemple, la peine maximale pour voies de fait (article 266) est de cinq ans d'emprisonnement, peu importe que l'infraction ait été ou non motivée en partie par la haine ${ }^{125}$.» Il conclut que l'article 718 «démontre le manque de fermeté du Canada quant à cette question. [...] Rien ne prouve " que la mise en vigueur des dispositions "a changé la façon dont les juges punissent les personnes condamnées pour un crime qu'elles ont commis sous l'emprise de la haine ${ }^{126} "$ (ibid : 32).

\section{L'alourdissement de la peine... efficace ou non?}

Le chef de l'unité des crimes motivés par la haine de la Police de 
Toronto semble corroborer l'analyse de Roberts (1999). Il m'a indiqué qu'il n'avait jamais vu un agresseur homophobe recevoir une peine plus sévère parce que l'article 718 avait été appliqué. Au cours de sa carrière, le seul cas où un agresseur a reçu une peine plus lourde pour un crime motivé par la haine est celui d'un skinhead qui avait attaqué un Noir dans un tramway en criant : «Le pouvoir aux Blancs!» À son arrestation, il avait un manifeste à propos racistes dans ses poches, et la police découvrit qu'il fréquentait des sites Web à caractère haineux. Plutôt que de recevoir une peine à purger dans la collectivité, il fut condamné à cinq mois d'emprisonnement ${ }^{127}$.

Dans le cas de l'agression sordide du dentiste Ed Pollak et de son conjoint qui eut lieu à Toronto, le juge refusa de reconnaître qu'il s'agissait d'un acte de violence contre les gais. En 1993, les deux hommes virent une voiture se diriger vers eux dans le quartier gai. Ils s'enlevèrent du chemin, mais quatre hommes sortirent de la voiture. Pollak eut la lèvre fendue à quatre endroits et dut se faire faire des points de suture. Il perdit également une dent et eut besoin d'un traitement de canal pour deux autres dents ${ }^{128}$. Son conjoint reçut 15 coups de botte à la tête ${ }^{129}$. Des témoins notèrent le numéro de plaque de la voiture, mais la police mit 45 minutes à arriver sur les lieux. Les agents de police ne cherchèrent pas à savoir si l'agression pouvait s'expliquer par l'homophobie des agresseurs et ne demandèrent même pas aux victimes si elles avaient besoin d'une ambulance. «Tout ce qu'ils voulaient, c'était partir de là », a affirmé Pollak ${ }^{130}$.

Un procès fut finalement intenté à trois jeunes hommes de la région de Hamilton. L'un d'eux admit avoir demandé aux victimes ce qu'elles regardaient, ce qui ne l'empêcha pas, lui et l'un de ses comparses, d'invoquer "la légitime défense". Pour sa part, le troisième accusé déclara qu'il avait tenté de calmer les choses. Un témoin affirma qu'il avait entendu quelqu'un crier «Pédés! " Malgré cela, l'accusé nia que son geste eût été motivé par l'homophobie ${ }^{131}$. Un autre témoin, qui promenait son chien, déclara qu'il avait vu " un homme se faire frapper et donner des coups de pied, alors qu'il était recroquevillé contre un mur pour tenter de se protéger ${ }^{132}$." 
Le juge admit que la haine pouvait avoir « joué un rôle » dans la perpétration de l'infraction; toutefois, il refusa de déclarer qu'il s'agissait d'un "acte de violence à l'endroit des homosexuels ${ }^{133}$." Seuls deux des trois hommes furent reconnus coupables : ils furent condamnés à six mois d'emprisonnement et à deux ans de probation. Cependant, les deux hommes furent libérés au bout de dix jours après avoir versé une caution de $10000 \$$. Le ministère public et la défense portèrent la décision en appel. Le juge de la Cour d'appel de l'Ontario était lui aussi d'avis que les victimes n'avaient pas été agressées en raison de leur orientation sexuelle. La sentence des deux accusés fut réduite, passant à six mois d'emprisonnement avec sursis sans période de probation ${ }^{134}$.

Pollak se suicida trois mois avant la tenue de l'audience d'appel. Peu de temps avant son décès, il avait déclaré «qu'une partie de sa liberté s'était envolée à jamais. [...] Le simple fait de parler de ce qui m'est arrivé m'a donné des forces ${ }^{135}$. " Un ancien coordonnateur du programme antiviolence de Toronto déclara : "Les dispositions qui autorisent l'alourdissement de la peine sont inutiles. Les juges ne veulent pas s'en servir. Je ne crois pas que le système de justice pénale réponde en aucune façon aux besoins des LGBT victimes de violence : ces victimes ne sont pas reconnues et on ne s'en soucie pas ${ }^{136}$."

\section{Recours judiciaires à la disposition des victimes}

Divers recours judiciaires s'offrent aux victimes des actes de violence envers les LGBT. Jeffery (1998) a rédigé un important manuel dans lequel il explique clairement aux Canadiens victimes de crimes motivés par la haine les nombreuses façons d'obtenir réparation pour les torts subis. Entre autres, en application du paragraphe 737(3) du Code criminel, le juge peut imposer au contrevenant, comme condition de sa remise en liberté, de verser un dédommagement. Malgré cette possibilité, une étude démontre que le dédommagement n'a été ordonné que six fois sur 4294 comparutions, ce qui représente un rapport de moins de $0,1 \% 137$. 
"...une étude

démontre que le

dédommagement

n'a été ordonné que

six fois sur 4294

comparutions, ce

qui représente un

rapport de moins de

0,1\%137.»
En plus des dispositions qui s'appliquent à des infractions manifestes (voies de fait, agression sexuelle, tentative de meurtre, etc.), d'autres articles peuvent être invoqués selon le cas :

- L'article portant sur la torture (article 269.1) prévoit l'imposition d'une peine pouvant aller jusqu'à 14 ans d'emprisonnement aux personnes qui, " avec le consentement d'un fonctionnaire ou à sa demande ", infligent intentionnellement à autrui " une douleur ou des souffrances aiguës, physiques ou mentales, afin de l'intimider, de faire pression sur lui, de le punir ou d'obtenir des renseignements de lui ou d'une tierce personne ${ }^{138}$."

- Lorsque les agresseurs agissent en bande, ils peuvent être accusés de harcèlement criminel (article 264), d'intimidation ou de surveillance (article 423).

- Quelqu'un qui fait des menaces au téléphone peut être accusé de propos indécents ou de harcèlement au téléphone (article 372) ou encore de profération de menaces (article 264.1).

- Le harcèlement au téléphone peut également être traité en application de l'article 13 de la Loi canadienne sur les droits de la personne $e^{139}$.

- Les tabasseurs chez qui « revient le même comportement de violence sexuelle ou physique» envers des homosexuels et dont les gestes « risquent d'entraîner le décès, des dommages corporels ou de graves préjudices psychologiques chez d'autres personnes " peuvent aussi être déclarés délinquants dangereux ${ }^{140}$.

Malheureusement, les victimes LBGT ne peuvent pas toujours se fier à l'État pour mener une enquête et mettre en accusation les meurtriers. Par exemple, je traite dans le chapitre suivant d'un cas où une douzaine de policiers de Montréal ont été filmés en train de battre des manifestants gais, sans qu'apparemment une accusation criminelle soit portée.

Dans certains cas, le seul moyen dont dispose la victime pour que justice soit faite est d'intenter une poursuite privée. Même s'il faut pour cela une grande expertise légale, en plus des ressources financières et du temps requis, cette démarche peut avoir un impact significatif. En effet, la décision rendue peut 
«En 1998, Jeffery souligna que le montant moyen accordé en Ontario était limité à $5000 \$ . »$ faire jurisprudence pour les litiges futurs, et il s'agit d'un moyen de faire réagir le public ${ }^{141}$. Dans cette perspective, des groupes juifs engagèrent une poursuite privée contre Ernest Zundel, l'accusant d'avoir " fait volontairement de fausses déclarations". L'État finit par se charger de la cause, qui s'est rendue jusqu'à la Cour suprême du Canada. L'affaire lança un grand débat public sur l'antisémitisme au Canada.

Les victimes de violence envers les LGBT peuvent également recevoir une indemnisation, mesure qui relève des provinces. Bien qu'il soit relativement facile d'obtenir un dédommagement, il y a de nombreux désavantages à le faire. En 1998, Jeffery souligna que le montant moyen accordé en Ontario était limité à 5000 \$. Le plafond de l'indemnité forfaitaire y était fixé à 25000 \$ et à $1000 \$$ en cas de versements périodiques. De plus, les victimes devaient présenter leur demande dans l'année suivant le crime, et il pouvait s'écouler jusqu'à trois ans avant que tout soit réglé, sans compter que l'indemnité reçue avait une incidence sur les prestations d'aide sociale, les produits d'assurance et la pension d'invalidité ${ }^{142}$.

Par ailleurs, les victimes peuvent s'adresser à des commissions quasi indépendantes pour porter plainte contre les forces policières. Dans certaines provinces, notamment en Ontario, ces commissions ont été abolies, et ce sont les chefs de police qui sont chargés de traiter les plaintes reçues. Dans le cas de Brian Nolan, exposé au chapitre précédent, des accusations finirent par être portées contre les agents de police de Terre-Neuve. Cependant, ces agents eurent comme unique sanction de «se faire taper sur les doigts. [...] L'un d'eux fut suspendu pendant 14 jours et un autre se vit infliger une suspension de dix jours. Ils ne jugèrent même pas bon de s'excuser ${ }^{143}$.»

Le montant maximal de l'indemnité accordée par les commissions des droits de la personne est généralement peu élevé : "Souvent, les défendeurs qui savent qu'il y a peu de chances que leur cause soit entendue par un tribunal ne tentent pas véritablement d'en arriver à un règlement, dans l'espoir que la commission n'aura pas les moyens de faire avancer le cas ${ }^{144}$." Les commissions provinciales et leur homologue fédéral encouragent 


"Le Tribunal
canadien
des droits de la
personne peut imposer
à l'intimé de verser
jusqu'à $20000 \$$
en compensation de
la douleur et des
souffrances subies par
la victime, en plus,
dans certains cas, de
devoir assumer les frais
encourus ${ }^{145} . "$

"Le Tribunal

des droits de la

personne peut imposer

à l'intimé de verser

jusqu'à 20000 \$

en compensation de

souffrances subies par

la victime, en plus,

dans certains cas, de

encourus $^{145}$." les deux parties à régler le litige par voie de médiation. Seule une fraction des plaintes sont traduites devant un tribunal, l'organe qui a le pouvoir d'établir les montants à verser et les autres mesures compensatoires. Le Tribunal canadien des droits de la personne peut imposer à l'intimé de verser jusqu'à 20000 \$ en compensation de la douleur et des souffrances subies par la victime, en plus, dans certains cas, de devoir assumer les frais encourus ${ }^{145}$.

Brian Nolan raconte, avec une certaine amertume, que la Commission des droits de la personne de Terre-Neuve et Labrador «s'est perdue dans les méandres des règles et de la bureaucratie. De plus, elle a décidé que mon dossier n'irait pas très loin. [...] Sept mois après l'agression, un agent de la Commission m'a téléphoné pour me dire que je n'avais pas présenté ma demande à temps et que celle-ci avait donc été classée sans suite. Pourtant, je m'étais présenté en personne au bureau le lundi suivant l'agression pour fournir les renseignements qu'il fallait. [...] Mon dossier s'est retrouvé sur les bureaux des uns et des autres. Au bout de deux ans, j'étais vraiment furieux. Je me disais : "Pourquoi me donner tant de mal alors qu'ils ne peuvent rien faire?” [...] Rendu à ce point, j'étais épuisé par toute l'affaire. À un moment donné, tu n'en peux plus ${ }^{146}$.»

De l'avis de Jeffery (1998), les litiges «entraînent des dépenses élevées, prennent beaucoup de temps et provoquent du stress chez les plaignants ». Même si les auteurs de crimes motivés par la haine sont le plus souvent des jeunes ayant des ressources financières limitées, les poursuites qui aboutissent à la condamnation des criminels peuvent placer ceux-ci dans une situation financière précaire ou les acculer à la faillite. Il en va de même des organisations qui, en l'absence de procès, ne seraient pas inquiétées en vertu des lois en vigueur. D'autre part, comme il s'agit de délits intentionnels, il n'est pas nécessaire de fournir de preuves des préjudices corporels subis : "La simple menace de préjudice suffit pour qu'il y ait agression. » Les poursuites "donnent aux victimes l'élan qui leur permet de prendre les choses en main et de cerner les enjeux; le fardeau de la preuve est moins onéreux et le plaignant peut recevoir des dommagesintérêts compensatoires et parfois punitifs ${ }^{147}$." 
"Les victimes de violence envers les LGBT peuvent également présenter un recours collectif."
Dans les cas de voies de fait, d'attaque en bande, d'agression sexuelle, d'intimidation et de harcèlement par téléphone, un certain nombre de motifs permettent d'intenter une poursuite au civil : négligence, voies de fait, coups, violation de propriété privée, infliction intentionnelle de souffrances morales et séquestration ${ }^{148}$. Cependant, il est rare que de gros montants soient accordés en dommages-intérêts punitifs dans le cas d'actes cruels, vindicatifs, répréhensibles et malfaisants. En 1996, il n'y avait eu dans l'histoire du Canada que quatre cas où le montant versé était supérieur à $100000 \$ \$^{149}$. De plus, afin d'éviter la double sanction, le versement de dommages-intérêts n'est pas de mise lorsque les contrevenants sont condamnés par une cour criminelle, sauf dans les cas où ils font l'objet d'une absolution sous condition.

Les victimes de violence envers les LGBT peuvent également présenter un recours collectif. Il s'agit « d'une poursuite judiciaire intentée par un représentant du groupe, en son nom et au nom d'autres personnes ayant subi le même préjudice, causé par le même acte ou la même omission. [...] Cette démarche permet à un grand nombre de victimes d'obtenir réparation pour le tort subi, alors qu'elles n'auraient pu se permettre d'intenter une poursuite par leurs propres moyens ${ }^{150}$."

Jeffery (1998) souligne ce qui suit : "Les recours collectifs demeurent un moyen que peu de gens comprennent et emploient, mais grâce auquel un groupe entier obtient justice dans des circonstances appropriées." Il s'agit essentiellement "d'une solution de rechange qui permet de faire appliquer la loi et d'obtenir une indemnisation privée " et qui a un effet dissuasif. En outre, ces recours forcent des personnes ou des organismes, qui normalement échappent à la loi, à subir une sanction économique. Toutefois, comme les procédures préliminaires sont onéreuses et exigent énormément de temps, seules les personnes qui sont en contact avec des avocats peuvent envisager cette solution. Cependant, la plupart des provinces consentent à verser une avance pour couvrir les coûts initiaux. 


\section{Conclusion}

Les recours juridiques énumérés justifient amplement la création d'un organisme national qui aiderait les victimes de violence motivée par l'homophobie à prendre des mesures contre leurs agresseurs, et aussi contre les acteurs du système judiciaire, les policiers et les institutions qui manquent à leur devoir de protection $^{151}$.

\section{Bibliographie}

Aird, Elizabeth (1994). «Queer Patrol Doesn't Have Limp Wrist, Only Needed Muscle», Vancouver Sun, 11 juin 1994, p. A3.

Amsden, Cynthia (1995). «Portrait of a Killer», The Ottawa Citizen, 26 avril 1995, p. B3.

Anderssen, Erin (2000). «I'm Got Very Good at It, Killer Says», The Globe and Mail, 24 mars 2000, p. A1, A8.

Aronson, Peter; David E. Rovella et Bob Van Voris (1988). «Jurors: A Biased, Independent Lot», National Law Journal, 2 novembre 1998, p. A1.

Associated Press (2003). "Gay Sex Ban Struck Down by US Supreme Court», Globe and Mail, 26 juin 2003.

Backer, Larry (1996). "Constructing a "Homosexual” for Constitutional Theory: Sodomy Narrative, Jurisprudence and Antipathy in the U.S. and Britain», Tulane Law Review, 71, décembre 1996, p. 563-592.

Barr, Doug (1994). «Hate Season Kickoff», Angles, mai 1994, p. 1.

Blanchfield, Mike (1994). "Ottawa Man Convicted in Beating of Gay Man», The Ottawa Citizen, 31 mars 1994, p. B7.

Blanchfield, Mike (1994 a). «Outcasts Formed Deadly Brotherhood of Doomed», The Ottawa Citizen, 21 juin 1994, p.A1.

Blanchfield, Mike (1994 b). «Murder Denials Just “tall tales”», The Ottawa Citizen, 9 décembre 1994, p. B7.

Blanchfield, Mike (1994 c). «Crown Claims "Sex” Angle in Steamer Trunk Killing», The Ottawa Citizen, 14 décembre 1994, p. D11.

Blanchfield, Mike (1994 d). «Jury Surprises Court with Acquittal in Steamer Trunk Killing», The Ottawa Citizen, 17 décembre 1994, p. A1.

Brown, Eleanor (1994). «Victim Takes on Police», Xtra!, 18 février 1994, p. 15.

Brown, Eleanor (1995). «Suspects Arrested», Xtra!, 20 août 1993, p. 12; Amsden, Cynthia, «Portrait of a Killer», The Ottawa Citizen, 26 avril 1995, p. B3.

Brown, Eleanor (1999). «Death Won't Affect Trial», Xtra!, 14 janvier 1999, p. 13. 
Cherry, Paul (2000). «I'm Guilty of Gay Killings: McGray», Montreal Gazette, 26 avril 2000, p. A1A2.

Cossman, Brenda (2003). «Sex Law», Capital Xtra, 19 juin 2003, p. 15.

Craig, Alisa (1995). «Gaybashing Case Goes to Court», Xtra!, 17 mars 1995, p. 13.

Craig, Alisa (1996). «A Bashing or a Simple Assault?», Xtra!, 23 mai 1996, p. 18.

Darroch, Wendy (1996). «Pair Who Beat Man Ordered to Write Essays on Gays», The Toronto Star, 31 juillet 1996, p. A18.

DeMara, Bruce (1994). «Don't Be Afraid, Gay-Bashing Victims Say», The Toronto Star, 23 octobre 1994, p. A15.

Drummie, Gretchen (1995). «Suspect in Gay attack Denies Kicks», Toronto Sun, 11 mars 1995, p. 28.

Drummie, Gretchen (1995 a). “"Booze, Machismo, Hate”: Prosecutor Explains Gay-Bashing», Toronto Sun, 16 mars 1995, p. 43.

Drummie, Gretchen (1995 b). «Attackers Get Jail», Toronto Sun, 13 mai 1995, p. 17.

Drummie, Gretchen (1995 c). «Man Acquitted in Gay Bashing», Toronto Sun, 28 octobre 1995, p. 28.

Garro, Julia (2004). «Armed and Dangerous», Xtra!, 22 janvier 2004, p. 13.

Giese, Rachel (1995). «Hating the Hate-Crime Bill», This Magazine, novembre 1995, p. 7-9.

Griffin, Kevin (1994 a). «Publicize Gay-Bashing, Activist Tells Police», Vancouver Sun, 14 mai 1994, p. A7.

Griffin, Kevin (1994). «Gays Fight Back after Attack in Coffee Bar», Vancouver Sun, 11 mai 1994, p.A2.

Hamilton, Graeme (2000). «The Torments That Sear the Soul of Michael McGray», The National Post, 24 mars 2000, p. A3.

Jacobs, James et Kimberly Potter (1998), Hate Crimes: Criminal Law and Identity Politics, Oxford University Press, New York.

Janoff, Douglas Victor (1995). Gay-bashing in Vancouver: A Case Study, non publié, 1995.

Jeffery, Bill (1998). Standing Up to Hate: Legal Remedies Available to Victims of Hate-Motivated Activity, Ottawa, ministère du Patrimoine canadien, 1998.

Jenness,Valerie et Ryken Grattet (1996). «The Criminalization of Hate: A Comparison of Structural and Policy Influences in the Passage of "Bias-Crime" Legislation in the United States», Sociological Perspectives, 39, 1, 1996, p. 129-154.

Jenness, Valerie (1999). «Managing Differences and Making Legislation: Social Movements, and the Racialization, Sexualization, and Gendering of Federal Hate Crime Law in the US», Social Problems, 46, 4, 1999, p. 549-561.

Jenness, Valerie (2001). «The Hate Crime Canon and Beyond: A Critical Assessment», Law and Critique, 12, 3, 2001, p. 293-294.

Jenness, Valerie et Ryken Grattet (2001), Making Hate a Crime: From Social Movement to Law Enforcement, Sage, New York.

Kinsman, Gary (2003). «History», Capital Xtra, 17 juillet 2003, p. 13.

Kirkby, Gareth (1998). «Police, Crown Give Run-Around », Xtra West, 12 novembre 1998, p. 10.

Leslie, Colin (1992). «Tenant Accused in Second Bashing», Xtra!, 3 avril 1992, p. 1. 
Leslie, Colin (1992 a). «Life Cut Short?», Xtra!, 17 avril 1992, p. 11.

Levin, Jack et Jack McDevitt (1993). Hate Crimes: The Rising Tide of Bigotry and Bloodshed, Plenum Press, New York, 1993.

Lofaro, Tony (1994). «Gatineau Death Suspect Nabbed by Ottawa RCMP», The Ottawa Citizen, 21 octobre 1994, p. B8.

MacDonal, Rory (2000). “"Gay Men Easy Targets for Murder”, Says Killer», Capital Xtra, 14 avril 2000, p. 13.

MacDougall, Bruce (1998). Queer Judgments: Homosexuality, Expression, and the Courts in Canada, University of Toronto Press, Toronto, 2000, p. 175; Bradley, Lara, «Educate the Enemy», Xtra!, 17 décembre 1998, p. 11.

MacDougall, Bruce (2000). Queer Judgments: Homosexuality, Expression, and the Courts in Canada, University of Toronto Press, Toronto, 2000, p. 259-260.

Maroney,T. (1993). «The Struggle against Hate Crime Movement at a Cross-roads», New York University Law Review, 73, 1993, p. 546-620.

Mason, Gail (2001). «Not Our Kind of Hate Crime», Law and Critique, 12, 3, 2001, p. 255-260.

Mennie, James (2003). «Montreal Judge Rules Swingers Clubs Legal, but Not Orgies», The Ottawa Citizen, 6 juillet 2003, p. G5.

Millar, Cal (1993). «Police Delve into Mystery Behind 3 Stabbing Deaths», The Toronto Star, 7 août 1993, p. A10.

Moloney, Paul (1993). «Gay Community Cautious after Two Men Beaten to Death», The Toronto Star, 4 août 1993, p. A6.

Nairne, Doug (1997). «Hate Mail Case Thrown Out», Winnipeg Free Press, 29 mai 1997, p. A1.

Presse canadienne (1996). "Judge Lets Potential Jurors Be Queried on Gay Biais», Canadian Press Newswire, 11 octobre 1996.

Presse canadienne (1999). «Gay-Basher in Court», Vancouver Province, 15 décembre 1999, p. A30.

Roberts, Julian (1999). Legislative Responses to Hate-Motivated Crime (rapport préliminaire du séminaire intitulé Domain Seminar on Social Justice du ministère du Patrimoine canadien), 14-15 mai 1999.

Robinson, Svend (2003). «Svend Robinson», The Globe and Mail, 27 mai 2003, téléchargé du site www.globeandmail.ca.

Robson, Ruthann (1995). «Convictions: Theorizing Lesbians and Criminal Justice»; Herman, Didi et Carl Stychin, éd., Legal Inversions: Lesbians, Gay Men and the Law, Temple University Press, Philadelphie, 1995, p. 191.

Rollason, Kevin (1994). «High Court Reduces Gay-Bash Sentence», The Winnipeg Free Press, 14 juin 1994.

Rosga,Annjanette (2001). «Deadly Words: State Power and the Entanglement of Speech andViolence in Hate Crime», Law and Critique, 12, 3, 2001, p. 239-240.

Ryder, Bruce (1991). «Straight Talk: Male Heterosexual Privilege», Queen's Law Journal, 16, p. $294-$ 295.

Shaffer, Martha (1995). "Criminal Responses to Hate-Motivated Violence», McGill Law Journal, 41, 1995, p. 210-246. 
Shahin, Mike (1995). «Escaper Gets Life in Prison for Murder of Montreal Man», The Ottawa Citizen, 18 janvier 1995, p. B2.

Sherrod, Drury et Peter Nardi (1998). «Homophobia in the Courtroom: An Assessment of Biases Against Gay Men and Lesbians in a Multiethnic Sample of Potential Jurors», dans Gregory Herek, éd., Stigma and Sexual Orientation: Understanding Prejudice against Lesbians, Gay Men and Bisexuals, Sage, Thousand Oaks, Californie, 1998, p. 24-25.

Still, Larry (1991). «Burnaby Woman Given Unusual Accessory Conviction», Vancouver Sun, 31 mai 1991, p. A1.

Stychin, Carl (1995). Law's Desire, Routledge, New York, 1995, p. 148.

Sumi, Glenn (1995). «Playing with the Truth», Xtra!, 18 août 1995, p. 32.

Takhar, Raj (1996). «Attack on Gays Draws Sentence», West End Times, 10 mai 1996, p. 7.

Takhar, Raj (1996 a). «Basher Pleads Guilty», Angles, mai 1996, p. 1.

Upton, Sean (1994). «Reaction of Dead Man's Lover “Odd”, Court told», The Ottawa Citizen, 26 mars 1994, p. C5.

Ward, Bruce (1993). «Man, Charged in Second Death, Accused in Friend's Slaying Insists: "I'm Not Gay."», The Ottawa Citizen, 7 août 1993, p. C1.

«History: Violence against the Gay/Lesbian/Transgendered Community», rapport rédigé par le coordonnateur des relations avec la communauté, Police régionale de Hamilton-Wentworth, 28 mai 1999.

\section{CAUSES :}

Bowers c. Hardwick (1986). 478 U.S., p. 186.

R. c. Brown [1993], 2 ALL ER 75 (HL).

R. c. Carolan [1995], 163 AR 238 (Alta. Prov Ct.) 239, parag. 8; entrevue avec un représentant du Bureau du procureur général, Calgary, 29 juin 1999.

R. c. Cvetan [1999], O.J. no 250.

R. c. Gendreau [1980], 33 Man. R., (2nd) 245 (CA), cité dans Donald Casswell, Lesbians, Gay Men and Canadian Law, Edmond Montgomery, Toronto, 1996, p. 616.

R. c. Jolicœur [1997], non rapporté, BC Prov. Ct., 7 février, cité dans Barriere, Garth, «Asking for Trouble», Xtra West, 18 septembre 1997, p.

R. c. McDonald [1995], OJ no 2137 (QL) (Ont. Prov. Ct. Gen. Div.); Pazzano, Sam, «Robber Who Preyed on Gay Men Jailed», Toronto Sun, 10 janvier 1995, p. 22.

R. c.Wilson [1990], 59 CCC (3rd) 432 (BCCA), cité dans ibid., p. 609.

R. c. M. (D.J.), [1990] OJ no 514 (QL) (Prov. Ct.-Youth Off. Ct.); appel rejeté, 61 CCC (3rd) 129 (OCA), cité dans MacDougall, Bruce, Queer Judgments: Homosexuality, Expression, and the Courts in Canada, University of Toronto Press, Toronto, 2000, p. 173.

\section{Notes}

${ }^{1}$ «Loi, homophobie et violence : légiférer pour punir les crimes motivés par la haine» est le chapitre IV du livre de Douglas Victor Janoff — Pink Blood - La violence homophobe au Canada, qui paraîtra cet automne aux Éditions Trityque. Ce livre a d'abord été édité en anglais 
sous le titre - Pink Blood - Homophobic Violence in Canada (University of Toronto Press, 2005). Le présent chapitre a été adapté pour sa publication dans Reflets, en y ajoutant toutes les références en bas de page.

${ }^{2}$ Griffin, Kevin, «Publicize Gay-Bashing, Activist Tells Police», Vancouver Sun, 14 mai 1994, p. A7.

${ }^{3}$ Griffin, Kevin, "Gays Fight Back after Attack in Coffee Bar», Vancouver Sun, 11 mai 1994, p. A2.

${ }^{4}$ Janoff, Douglas Victor, Gay-bashing in Vancouver: A Case Study, non publié, 1995, p. 6.

${ }^{5}$ Griffin, Kevin, "Gays Fight Back after Attack in Coffee Bar», Vancouver Sun, 11 mai 1994, p. A2.

${ }^{6}$ Aird, Elizabeth, «Queer Patrol Doesn't Have Limp Wrist, Only Needed Muscle», Vancouver Sun, 11 juin 1994, p. A3.

${ }^{7}$ Barr, Doug, «Hate Season Kickoff», Angles, mai 1994, p. 1.

${ }^{8}$ Janoff, Douglas Victor, Gay-bashing in Vancouver: A Case Study, non publié, 1995, p. 7-8.

${ }^{9}$ Ibid., p. 6.

${ }^{10}$ Ibid., p. 10-11.

${ }^{11}$ Ibid., p. 8-9.

${ }^{12}$ Takhar, Raj, «Attack on Gays Draws Sentence», West End Times, 10 mai 1996, p. 7.

${ }^{13}$ Takhar, Raj, «Basher Pleads Guilty», Angles, mai 1996, p. 1.

${ }^{14}$ Ryder, Bruce, «Straight Talk: Male Heterosexual Privilege», Queen's Law Journal, 16, 1991, p. 294-295.

15 Robson, Ruthann, "Convictions: Theorizing Lesbians and Criminal Justice»; Herman, Didi et Carl Stychin, éd., Legal Inversions: Lesbians, Gay Men and the Law, Temple University Press, Philadelphie, 1995, p. 191.

${ }^{16}$ Stychin, Carl, Law's Desire, Routledge, New York, 1995, p. 148.

${ }^{17}$ R. c. Brown [1993], 2 ALL ER 75 (HL), cité dans ibid., p. 129.

${ }^{18}$ Ibid., p. 137.

${ }^{19}$ Ibid., p. 102-103.

${ }^{20}$ Backer, Larry, "Constructing a "Homosexual" for Constitutional Theory: Sodomy Narrative, Jurisprudence and Antipathy in the U.S. and Britain», Tulane Law Review, 71, décembre 1996, p. 563-564.

${ }^{21}$ Associated Press, «Gay Sex Ban Struck Down by US Supreme Court», Globe and Mail, 26 juin 2003, www.globeandmail.ca/servlet/story/RTGAM.20030626.wgsex0626/BNStory.html.

${ }^{22}$ Bowers c. Hardwick, 478 U.S., 1986, p. 186.

${ }^{23}$ Backer, Larry, "Constructing a "Homosexual" for Constitutional Theory: Sodomy Narrative, Jurisprudence and Antipathy in the U.S. and Britain», Tulane Law Review, 71, décembre 1996, p. 592.

${ }^{24}$ Kinsman, Gary, «History», Capital Xtra, 17 juillet 2003, p. 13.

${ }^{25}$ Cossman, Brenda, «Sex Law», Capital Xtra, 19 juin 2003, p. 15.

${ }^{26}$ Mennie, James, «Montreal Judge Rules Swingers Clubs Legal, but Not Orgies», The Ottawa Citizen, 6 juillet 2003, p. G5.

${ }^{27}$ Presse canadienne, «Judge Lets Potential Jurors Be Queried on Gay Biais», Canadian Press Newswire, 11 octobre 1996.

${ }^{28}$ Aronson, Peter, David E. Rovella et Bob Van Voris, «Jurors: A Biased, Independent Lot», National Law Journal, 2 novembre 1998, p. A1. 
${ }^{29}$ Blanchfield, Mike, «Murder Denials Just “tall tales”», The Ottawa Citizen, 9 décembre 1994, p. B7.

${ }^{30}$ Blanchfield, Mike, «Jury Surprises Court with Acquittal in Steamer Trunk Killing», The Ottawa Citizen, 17 décembre 1994, p.A1.

${ }^{31}$ Blanchfield, Mike, "Crown Claims "Sex" Angle in Steamer Trunk Killing», The Ottawa Citizen, 14 décembre 1994, p. D11.

32 Blanchfield, Mike, "Murder Denials Just "tall tales"», The Ottawa Citizen, 9 décembre 1994, p. B7.

${ }^{33}$ R. c. Gendreau [1980], 33 Man. R., (2nd) 245 (CA), cité dans Donald Casswell, Lesbians, Gay Men and Canadian Law, Edmond Montgomery, Toronto, 1996, p. 616.

${ }^{34}$ R. c. Wilson [1990], 59 CCC (3rd) 432 (BCCA), cité dans ibid., p. 609.

${ }^{35}$ MacDougall, Bruce, Queer Judgments: Homosexuality, Expression, and the Courts in Canada, University of Toronto Press, Toronto, 2000, p. 259-260.

${ }^{36}$ R. c. Jolicœur [1997], non rapporté, BC Prov. Ct., 7 février, cité dans Barriere, Garth, «Asking for Trouble», Xtra West, 18 septembre 1997, p. 17.

${ }^{37}$ R. c. M. (D.J.), [1990] OJ no 514 (QL) (Prov. Ct.-Youth Off. Ct.); appel rejeté, 61 CCC (3rd) 129 (OCA), cité dans MacDougall, Bruce, Queer Judgments: Homosexuality, Expression, and the Courts in Canada, University of Toronto Press, Toronto, 2000, p. 173.

${ }^{38}$ Leslie, Colin, «Life Cut Short?», Xtra!, 17 avril 1992, p. 11.

${ }^{39}$ Entrevue auprès de militants locaux, 13 juillet 1999.

${ }^{40}$ MacDougall, Bruce, Queer Judgments: Homosexuality, Expression, and the Courts in Canada, University of Toronto Press, Toronto, 2000, p. 175; Bradley, Lara, «Educate the Enemy», Xtra!, 17 décembre 1998, p. 11.

${ }^{41}$ Entrevue, 26 mai 1999.

${ }^{42}$ Presse canadienne, «Gay-Basher in Court», Vancouver Province, 15 décembre 1999, p. A30.

${ }^{43}$ Leslie, Colin, «Tenant Accused in Second Bashing», Xtra!, 3 avril 1992, p. 1; Leslie, Colin, "Accused Basher Banned», Xtra!, 17 avril 1992, p. 11.

${ }^{44}$ Darroch, Wendy, «Pair Who Beat Man Ordered to Write Essays on Gays», The Toronto Star, 31 juillet 1996, p. A18; Craig, Alisa, «A Bashing or a Simple Assault?», Xtra!, 23 mai 1996, p. 18.

45 Rollason, Kevin, «High Court Reduces Gay-Bash Sentence», The Winnipeg Free Press, 14 juin 1994.

46 R. c. Gallant [1994], 95 Man. R. (2nd) 296 (CA); MacDougall, Bruce, Queer Judgments: Homosexuality, Expression, and the Courts in Canada, University of Toronto Press, Toronto, 2000, p. 174.

${ }^{47}$ Lettre, 13 avril 1999.

${ }^{48}$ Entrevue avec un agent de la Police de Vancouver, 13 mai 1999.

${ }^{49}$ Kirkby, Gareth, «Police, Crown Give Run-Around », Xtra West, 12 novembre 1998, p. 10.

${ }^{50}$ Entrevue avec un agent de la Police de Vancouver, 13 mai 1999.

${ }^{51}$ DeMara, Bruce, «Don’t Be Afraid, Gay-Bashing Victims Say», The Toronto Star, 23 octobre 1994, p.A15.

${ }^{52}$ Drummie, Gretchen, «Man Acquitted in Gay Bashing», Toronto Sun, 28 octobre 1995, p. 28. 
${ }^{53}$ Sumi, Glenn, «Playing with the Truth», Xtra!, 18 août 1995, p. 32.

54 «History:Violence against the Gay/Lesbian/Transgendered Community», rapport rédigé par le coordonnateur des relations avec la communauté, Police régionale de Hamilton-Wentworth, 28 mai 1999.

${ }^{55}$ Garro, Julia, «Armed and Dangerous», Xtra!, 22 janvier 2004, p. 13.

${ }^{56}$ R. c. McDonald [1995], OJ no 2137 (QL) (Ont. Prov. Ct. Gen. Div.); Pazzano, Sam, «Robber Who Preyed on Gay Men Jailed», Toronto Sun, 10 janvier 1995, p. 22.

${ }^{57}$ Still, Larry, «Burnaby Woman Given Unusual Accessory Conviction», Vancouver Sun, 31 mai 1991, p.A1.

${ }^{58}$ Upton, Sean, «Reaction of Dead Man's Lover “Odd”, Court told», The Ottawa Citizen, 26 mars 1994, p. C5.

${ }^{59}$ Blanchfield, Mike, «Ottawa Man Convicted in Beating of Gay Man», The Ottawa Citizen, 31 mars 1994, p. B7.

${ }^{60}$ R. c. Carolan [1995], 163 AR 238 (Alta. Prov Ct.) 239, parag. 8; entrevue avec un représentant du Bureau du procureur général, Calgary, 29 juin 1999.

${ }^{61}$ Ibid.

${ }^{62}$ Entrevue, $1^{\text {er }}$ juillet 1999.

${ }^{63}$ Lofaro, Tony, "Gatineau Death Suspect Nabbed by Ottawa RCMP», The Ottawa Citizen, 21 octobre 1994, p. B8.

${ }^{64}$ Shahin, Mike, «Escaper Gets Life in Prison for Murder of Montreal Man», The Ottawa Citizen, 18 janvier 1995, p. B2.

${ }^{65}$ Lofaro, Tony, «Gatineau Death Suspect Nabbed by Ottawa RCMP», The Ottawa Citizen, 21 octobre 1994, p. B8.

${ }^{66}$ Shahin, Mike, «Escaper Gets Life in Prison for Murder of Montreal Man», The Ottawa Citizen, 18 janvier 1995, p. B2.

${ }^{67}$ Lofaro, Tony, «Gatineau Death Suspect Nabbed by Ottawa RCMP», The Ottawa Citizen, 21 octobre 1994, p. B8.

${ }^{68}$ Shahin, Mike, «Escaper Gets Life in Prison for Murder of Montreal Man», The Ottawa Citizen, 18 janvier 1995, p. B2.

${ }^{69}$ Brown, Eleanor, «Suspects Arrested», Xtra!, 20 août 1993, p. 12; Amsden, Cynthia, «Portrait of a Killer», The Ottawa Citizen, 26 avril 1995, p. B3.

${ }^{70}$ Moloney, Paul, «Gay Community Cautious after Two Men Beaten to Death», The Toronto Star, 4 août 1993, p. A6.

${ }^{71}$ Millar, Cal, «Police Delve into Mystery Behind 3 Stabbing Deaths», The Toronto Star, 7 août 1993, p.A10.

${ }^{72}$ Amsden, Cynthia, «Portrait of a Killer», The Ottawa Citizen, 26 avril 1995, p. B3.

${ }^{73}$ Moloney, Paul, «Gay Community Cautious after Two Men Beaten to Death», The Toronto Star, 4 août 1993, p. A6.

${ }^{74}$ «Warrants Issued», The Globe and Mail, 4 août 1993, p. A10.

${ }^{75}$ Millar, Cal, «Police Delve into Mystery Behind 3 Stabbing Deaths», The Toronto Star, 7 août 1993, p.A10. 
${ }^{76}$ Blanchfield, Mike, «Outcasts Formed Deadly Brotherhood of Doomed», The Ottawa Citizen, 21 juin 1994, p. A1.

${ }^{77}$ Amsden, Cynthia, «Portrait of a Killer», The Ottawa Citizen, 26 avril 1995, p. B3.

${ }^{78}$ Ward, Bruce, «Man, Charged in Second Death,Accused in Friend's Slaying Insists: "I'm Not Gay.”", The Ottawa Citizen, 7 août 1993, p. C1.

${ }^{79}$ Blanchfield, Mike, «Outcasts Formed Deadly Brotherhood of Doomed», The Ottawa Citizen, 21 juin 1994, p.A1.

${ }^{80}$ Amsden, Cynthia, «Portrait of a Killer», The Ottawa Citizen, 26 avril 1995, p. B3.

${ }^{81}$ Hamilton, Graeme, «The Torments That Sear the Soul of Michael McGray», The National Post, 24 mars 2000 , p. A3.

${ }^{82}$ Anderssen, Erin, «I'm Got Very Good at It, Killer Says», The Globe and Mail, 24 mars 2000, p. A1, A8.

${ }^{83}$ MacDonal, Rory, «"Gay Men Easy Targets for Murder”, Says Killer», Capital Xtra, 14 avril 2000, p. 13.

${ }^{84}$ Cherry, Paul, «I'm Guilty of Gay Killings: McGray», Montreal Gazette, 26 avril 2000, p. A1-A2.

${ }^{85}$ Jenness, Valerie, «Managing Differences and Making Legislation: Social Movements, and the Racialization, Sexualization, and Gendering of Federal Hate Crime Law in the US», Social Problems, 46, 4, 1999, p. 549.

${ }^{86}$ Mason, Gail, «Not Our Kind of Hate Crime», Law and Critique, 12, 3, 2001, p. 255-260.

${ }^{87}$ Rosga, Annjanette, «Deadly Words: State Power and the Entanglement of Speech andViolence in Hate Crime", Law and Critique, 12, 3, 2001, p. 239-240.

${ }^{88}$ Jacobs, James et Kimberly Potter, Hate Crimes: Criminal Law and Identity Politics, Oxford University Press, New York, 1998, p. 45-46.

${ }^{89}$ Levin, Jack et Jack McDevitt, Hate Crimes: The Rising Tide of Bigotry and Bloodshed, Plenum Press, New York, 1993.

${ }^{90}$ Jacobs, James et Kimberly Potter, Hate Crimes: Criminal Law and Identity Politics, Oxford University Press, New York, 1998, p. 53.

91 Jenness, Valerie, «Managing Differences and Making Legislation: Social Movements, and the Racialization, Sexualization, and Gendering of Federal Hate Crime Law in the US», Social Problems, 46, 4, 1999, p. 559-561.

92 Ibid., p. 553.

${ }^{93}$ Ibid., p. 557-559.

94 Jenness, Valerie, «The Hate Crime Canon and Beyond: A Critical Assessment», Law and Critique, 12, 3, 2001, p. 283-285.

95 Jenness, Valerie et Ryken Grattet, «The Criminalization of Hate: A Comparison of Structural and Policy Influences in the Passage of "Bias-Crime" Legislation in the United States», Sociological Perspectives, 39, 1, 1996, p. 129-154.

${ }^{96}$ Jenness, Valerie, «The Hate Crime Canon and Beyond: A Critical Assessment», Law and Critique, 12, 3, 2001, p. 286-287.

97 Jenness, Valerie et Ryken Grattet, Making Hate a Crime: From Social Movement to Law Enforcement, Sage, New York, 2001, p. 1-3.

98 Ibid., p. 6-7. 
${ }^{99}$ Ibid., p. 103-106.

100 Jenness, Valerie, «The Hate Crime Canon and Beyond: A Critical Assessment», Law and Critique, 12, 3, 2001, p. 293-294.

101 Jenness, Valerie et Ryken Grattet, Making Hate a Crime: From Social Movement to Law Enforcement, Sage, New York, 2001, p. 112.

102 Ibid., p. 117.

${ }^{103}$ Ibid., p. 147-151.

${ }^{104}$ Roberts, Julian, Legislative Responses to Hate-Motivated Crime (rapport préliminaire du séminaire intitulé Domain Seminar on Social Justice du ministère du Patrimoine canadien), 14-15 mai 1999, p. 4.

105 «Kill Fags», Xtra!, 9 juin 1995, p. 18.

${ }^{106}$ Nairne, Doug, «Hate Mail Case Thrown Out», Winnipeg Free Press, 29 mai 1997, p. A1.

107 Robinson, Svend, «Svend Robinson», The Globe and Mail, 27 mai 2003, téléchargé du site www.globeandmail.ca.

${ }^{108}$ Egale Canada, «Email update», 11 mai 2004.

109 Roberts, Julian, Legislative Responses to Hate-Motivated Crime (rapport préliminaire du séminaire intitulé Domain Seminar on Social Justice du ministère du Patrimoine canadien), 14-15 mai 1999 , p. 4.

${ }^{110}$ Ibid., p. 18.

111 Ibid., p. 12-13.

112 Ibid., p. 19.

${ }^{113}$ Ibid., p. 6-7.

114 Ibid., p. 24.

115 Ibid., p. 23.

${ }^{116}$ Maroney, T., «The Struggle against Hate Crime Movement at a Cross-roads», New York University Law Review, 73, 1993, p. 546-620.

${ }^{117}$ Giese, Rachel, «Hating the Hate-Crime Bill», This Magazine, novembre 1995, p. 7-9.

118 Jeffery, Bill, Standing Up to Hate: Legal Remedies Available toVictims of Hate-Motivated Activity, Ottawa, ministère du Patrimoine canadien, 1998, p. 21.

${ }^{119}$ Ibid., p. 213-214.

${ }^{120}$ Ibid., p. 245-246.

${ }^{121}$ Ibid., p. 203.

${ }^{122}$ Ibid., p. 245-246.

${ }^{123}$ Roberts, Julian, Legislative Responses to Hate-Motivated Crime (rapport préliminaire du séminaire intitulé Domain Seminar on Social Justice du ministère du Patrimoine canadien), 14-15 mai 1999, p. 19.

${ }^{124}$ Ibid., p. 32.

${ }^{125}$ Entrevue, 28 novembre 2002.

${ }^{126}$ Brown, Eleanor, «Victim Takes on Police», Xtra!, 18 février 1994, p. 15.

${ }^{127}$ Drummie, Gretchen, «Suspect in Gay attack Denies Kicks», Toronto Sun, 11 mars 1995, p. 28.

${ }^{128}$ Brown, Eleanor, «Victim Takes on Police», Xtra!, 18 février 1994, p. 15.

129 Drummie, Gretchen, «"Booze, Machismo, Hate”: Prosecutor Explains Gay-Bashing», Toronto Sun, 16 mars 1995, p. 43. 
${ }^{130}$ Craig, Alisa, «Gaybashing Case Goes to Court», Xtra!, 17 mars 1995, p. 13.

${ }^{131}$ Drummie, Gretchen, «Attackers Get Jail», Toronto Sun, 13 mai 1995, p. 17.

132 R. c. Cvetan [1999], O.J. no 250.

${ }^{133}$ Brown, Eleanor, «Death Won’t Affect Trial», Xtra!, 14 janvier 1999, p. 13.

${ }^{134}$ Entrevue avec un ancien coordonnateur, 26 mai 1999.

${ }^{135}$ Jeffery, Bill, Standing Up to Hate: Legal Remedies Available toVictims of Hate-Motivated Activity, Ottawa, ministère du Patrimoine canadien, 1998, p. 38-40.

${ }^{136}$ Ibid., p. 25.

${ }^{137}$ Ibid., p. 15.

${ }^{138}$ Ibid., p. 25.

139 Ibid., p. 17-19.

${ }^{140}$ Ibid., p. 28.

${ }^{141}$ Entrevue, 7 juillet 1999.

${ }^{142}$ Jeffery, Bill, Standing Up to Hate: Legal Remedies Available toVictims of Hate-Motivated Activity, Ottawa, ministère du Patrimoine canadien, 1998, p. 33.

${ }^{143}$ Entrevue avec un agent de la Commission canadienne des droits de la personne, 13 août 2003.

${ }^{144}$ Entrevue, 7 juillet 1999.

145 Jeffery, Bill, Standing Up to Hate: Legal Remedies Available toVictims of Hate-Motivated Activity, Ottawa, ministère du Patrimoine canadien, 1998, p. 38-40.

146 Ibid., p. 13-16.

${ }^{147}$ Ibid., p. 43.

148 Ibid., p. 43-44.

149 Ibid., p. 43-47. 